\title{
A Comprehensive Review on Tunnel Field-Effect Transistor (TFET) Based Biosensors: Recent Advances and Future Prospects on Device Structure and Sensitivity
}

\author{
N. Nagendra Reddy ${ }^{1} \cdot$ Deepak Kumar Panda ${ }^{1}$ \\ Received: 25 March 2020 / Accepted: 12 August 2020 / Published online: 26 August 2020 \\ (C) Springer Nature B.V. 2020
}

\begin{abstract}
In this fast-growing technological world biosensors become more substantial in human life and the extensive use of biosensors creates enormous research interest among researchers to define different approaches to detect biomolecules. The FET based biosensors have gained a lot of attention among all because of its high detection ability, low power, low cost, label-free detection of biomolecules, and CMOS compatible on-chip integration. The sensitivity of the biosensor inversely proportional to device size since they detect low concentration yields quick response time. Although FET based biosensor is having a lot of advantages among others but the short channel effects (SCE's) and the theoretical limitation on the subthreshold swing (SS $>60 \mathrm{mv} / \mathrm{dec}$ ) of the FET leads to restrict device sensitivity and also have higher power dissipation due to the thermionic emission of electrons. To avoid these problems researchers focus shifts to the new technology FET based biosensors i.e. TFET based biosensors which are having low power and superior characteristics due to Band to band tunneling of carrier and steep subthreshold swing. This manuscript describes the full-fledged detail about the TFET based biosensors right from unfolding the device evaluation to biosensor application which includes qualitative and quantitative parameters analysis study like sensitivity parameters and different factors affecting the sensitivity by comparing different structures and the mechanisms involved. The manuscript also describes a brief review of different sensitivity parameters and improvement techniques. This manuscript will give researchers a brief idea for developing for the future generation TFET biosensors with better performance and ease of fabrication.
\end{abstract}

Keywords Biosensor $\cdot$ Nanowire $\cdot$ Sensitivity $\cdot$ TFET $\cdot$ Tunneling

\section{Introduction}

The life-threatening bells of humans are at high alert because of the bio-attack that observed form the last few decades' right forms the HIV to present Coronavirus. They are invisible and spreading with lightning speed without the knowledge of humans and made their life so miserable. Apart from this, the technology improvement has given way for the replacement of classical warheads with bio warheads giving scope for the bio wars. These bio warheads/weapons consist of pathogenic virus or bacteria which spread very silently and took the lives of innocent people at the cutting edge. In this fastest growing technological world, the detection of biohazards

Deepak Kumar Panda

deepakiitkgp04@gmail.com; d.panda.2016@ieee.org

1 Microelectronics and VLSI Design Group, School of Electronics, VIT-AP University, Amaravati, Andhra Pradesh 522237, India (toxic gas of substance) becomes a challenge to every nation and the biosensors are given breakthroughs for this problem by giving a systematic approach for detection of the biomolecule. Because of its ideal characteristics, biosensors spread their applications in many areas like medical filed for earlystage detection and diagnosis [1], drug delivery, food processing, and environment monitoring, security and surveillance.

The biosensor is a device which can generates electrical signal form physiochemical reaction of biomolecules [2]. The sensing mechanism of the targeted biomolecule mainly consists of two different stages such as detection of the biomolecules and transduction. The detection stage carried out by analyzing the targeted biomolecules and in the transduction stage coverts this physiochemical reaction into measurable electrical which can be further processed.

After the discovery of first enzyme based biosensor by Clark et al. in 1962 [3], this emerging filed has gained a lot of attentions among worldwide researchers for developing accurate and reliable biosensors. Many researchers were 
reported wide variety of biosensors for different applications for fast, accurate and label free detection.

\section{Types of Biosensors}

Fundamentally Biosensors are classified by considering their detection mechanism and transduction method. The Detection mechanism involves the use of biological elements such as enzymes, biological tissues, antibodies, drugs, proteins, and microorganisms, etc. [2]. The targeted biomolecules analyzed by overlaying on the detection element as a result they generate some physiochemical reactions which generate some byproducts which are treated as inputs for the transducer elements. Depending upon the transduction process of different physiochemical reactions caused by the sensing elements are classified into four [4] major types and some subclass as given in the Fig. 1 [4].

All the biosensor mentioned in the above Fig. 4 provides the way for detection of a wide variety of biomolecules. The principle of operation of electrochemical biosensor is to experience the change in the electrical properties of the sensor from the reaction of the target biomolecules. The change observed is used as the measuring parameter for the sensor and based on parameter observed thy classified in to three different types as conductrometric, potentiometric and amperometric. The electrochemical biosensor detects different kinds of the biomolecules in human body like protein, biotin, uricase, DNA, glucose and haemoglobin and etc.

Optical biosensors are very powerful alternate for the conventional analytical type biosensors because it requires limited sample preparation for detection of target biomolecules.

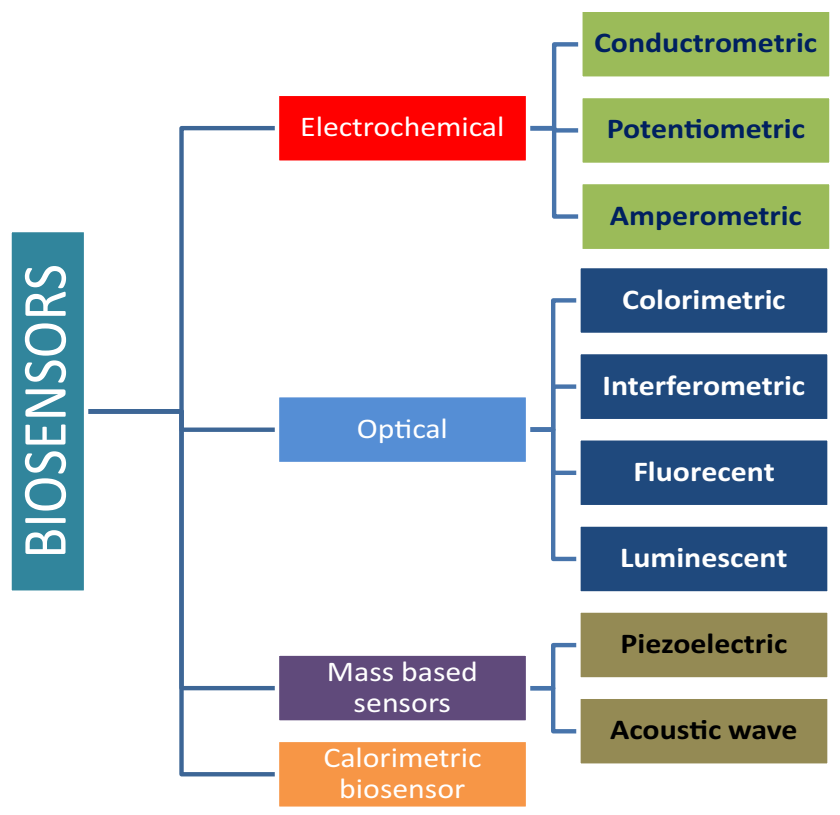

Fig. 1 Classification of biosensors based on the transducer [4]
Optical biosensor uses the interaction of the optical fields with the analyte for detection of biomolecules like tumour biomarkers, tumour cells and toxins etc. The mass based biosensors uses the basic principal of a response to change in mass. These sensors are takes major application in the MEMS devices specifically the piezoelectric base sensor is attracted lot of attention. The piezoelectric and the acoustic wave sensor come under these category and they are find very good application for the detection of DNA and glucose and living organisms. All kinds of biosensor are utilized for creating enhancement in the human life.

Basically for developing any accurate and reliable biosensor three main parameters should be considered such as sensitivity, specificity, and ease of fabrication. Among all kinds of biosensor electrochemical and optical biosensors are taken more attention because of their high specificity and low detection limit. The designs of mass-based and calorimetric biosensors are highly complicated and low response time. In the electrochemical biosensors potentiometric type transducer become more popular after the introduction of FET type biosensor because of high performance and low cost of fabrication.

\section{FET Based Biosensors}

In recent times FET based biosensors are gained a lot of attention among worldwide researchers due to their superior properties like label-free detection, small in size, rapid response, and reliability [6-12], the possibility of on-chip integration for amplification circuitry and sensor, mass production with low cost, high selectivity and reusability. To detect targeted biomolecules the oxide layer of the FET is employed with the bio receptors/bio-recognition element. Once these receptors captured the targeted biomolecules they have undergone conjugation process which generates electrochemical reactions and these electrochemical reactions lead to the gating effect of the semiconductor device [5, 13]. This gating effect changes the electrical properties of the device and characterized as the sensitivity parameters for the detection of biomolecules before and after capturing the targeted biomolecules by the receptors. There are many parameters with which we can measure the sensitivity like current ratios (Ion/Ioff), the shift in threshold voltage (VT), the variation of ON current (Ion). Although FET based biosensors are having a lot of advantages among others but they are facing major issues like I. The scaling difficulties and the short channel effects (SEC'S) experienced by the FET in the process of miniaturization [7, 14]. II. The theoretical limitation on the minimum achievable subthreshold swing ( $\mathrm{SS}>60 \mathrm{mv} / \mathrm{dec}$ ) [7, 14]. All these issues lead to narrowing the device performance and sensitivity and, the thermionic emission of electron in FET results in high power dissipation. To avoid these problems researchers focus shifts to the new technology FET based biosensors i.e TFET based 
biosensors which are having low power and superior characteristics [7, 8, 15-18] due to Band to band tunneling of carrier and steep subthreshold swing. Another crucial measurable parameter of biosensors is the response time and to have a quick response the subthreshold swing should be as low as possible. Since the TFET can achieve the $\mathrm{SS}(\mathrm{SS}<60 \mathrm{mv} / \mathrm{dec})$ less than CFET, so recently a lot of research is going on for designing TFET based biosensors. The complete detail about the FET based biosensor is available in many literature surveys and research articles [19].

Currently there is a lot of progress in the development of TFET based biosensors but we are unable to get complete information regarding TFET based biosensors at one point. So in this manuscript for the first time we present the complete review on the TFET based biosensor at one place. This review carried out in four sections. Section-1 elaborated about the structure and working principle of the TFET as a biosensor. Section-2 describes the comparison between different kinds of available structures for the detection of biomolecules. Section-3describes the performance comparison of different biosensors in terms of sensitivity. Finally, the conclusion is drawn in Section-4.

\subsection{Structure and Working of the TFET as a Biosensor}

The basic structure of TFET consists of three regions the source, drain, and channel. The source and drain doping is the major difference that can be observed among TFET and Conventional Field Effect Transistor (CFET). In the CFET source and drain are doped with similar kinds of doping elements either P-type or N-type but in the case of TFET dissimilar doping done for both source and drain. The channel region is usually intrinsic or lightly doped in TFET. The structure resembles a $\mathrm{p}-\mathrm{i}-\mathrm{n}$ diode with a gate. Barrier width of TFET is made thin to allow the tunneling of the charge carriers (doping source and drain are very high for the possibility of tunneling at the barrier junctions and) mostly tunneling of the charge carrier occurs at the source-channel junction because the source is highly doped than drain.

The current characteristic of transistor describes its behavior under various biasing conditions and The TFET based biosensor has three electrodes i.e gate, drain, and the source such that the region between source and drain (i.e channel) equipped with a biorecognition element. This biorecognition element interacts with the targeted biomolecules and senses their presence and monitor electrical activity. The biosensor then directly transforms the biological information into a measurable signal. The operation of TFET based biosensor is summarized as 1) change in the concentration of charge at the surface of the channel(2) this change in the charge leads to the change in the effective gate voltage(gating effect) (3) the increment in the drain current because of reduction of effective tunneling length due to gating effect. Form Fig. 2 it is observed that before capturing the biomolecules the energy states of source and channel are not aligned but figure describe the effective bending of the energy bands giving scope for the tunneling.

The review begins with the comparison of different TFET based biosensors device structures and different techniques for improvement of sensitivity variation followed by the various analytical models developed in different works of literature.

\subsection{Different Structures of Available TFET Based Biosensor}

\subsubsection{Silicon Nanowire Based TFET Biosensor (SiNWTFET)}

Nanowire structures are preferred for TFET sensors since they provide good electrostatic gate control over the channel due to the small dimension and produce higher tunneling current. After exploring the electrical characteristic of the TFET device [15-18] researchers started to utilize these characteristics for the development of biosensors. In the year 2012 Deblina et al. proposed a Silicon nanowire-based TFET [SiNWTFET]

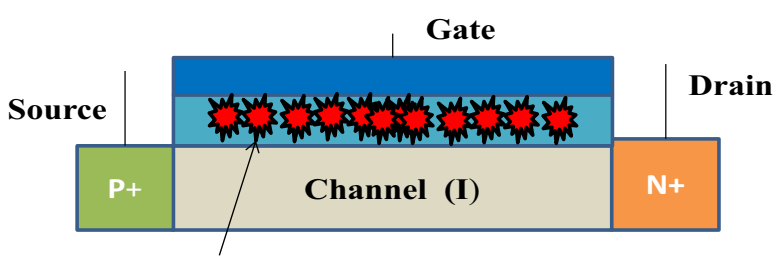

Targeted biomolecules

(a)

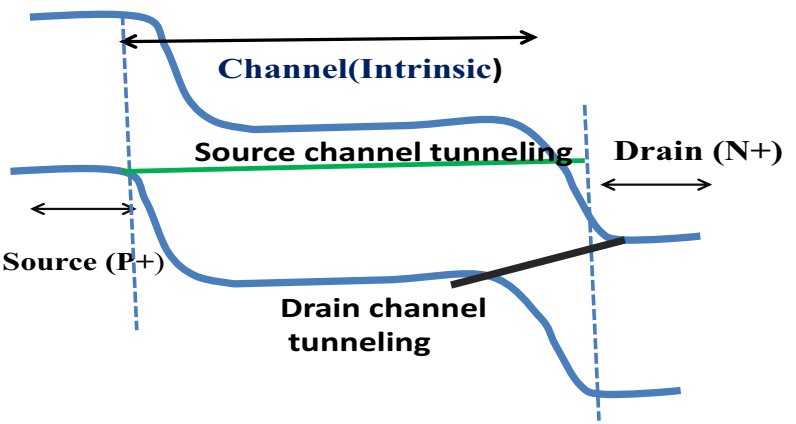

(b)

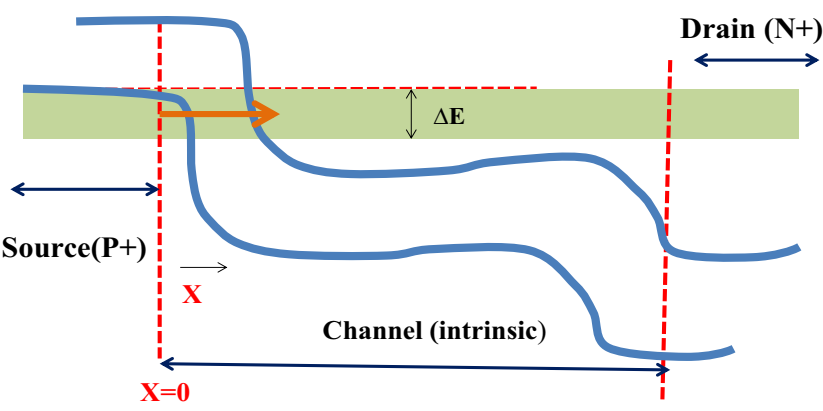

(c)

Fig. 2 a 2D structure of TFET (b) Energy band diagram in Off state (c) Energy band diagram in On state [20] 
Fig. 3 a Schematic arrangement nanowire based TFET biosensor. b Improved sensitivity as a function of biomolecules concentration [Fig. 1 and 3(c) [13]]

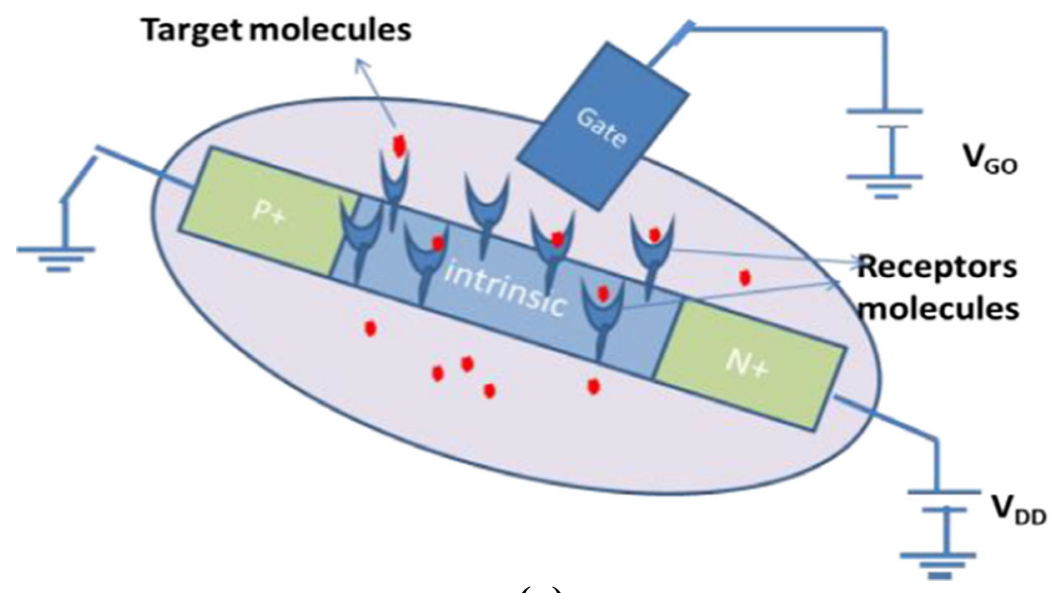

(a)

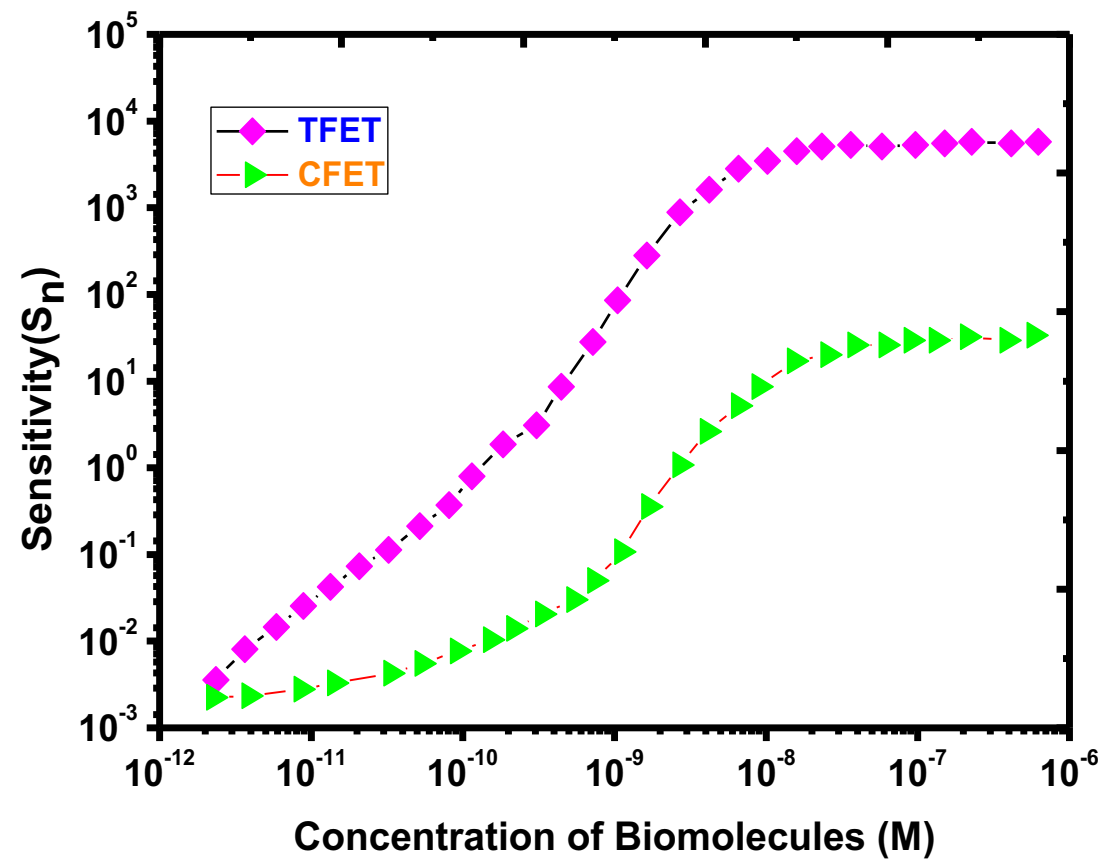

(b) biosensor for ultrasensitive and label-free detection [13] of biomolecules by keeping all the advantages of CFET biosensors. The structure of the biosensor utilizes a single nanowire to form the $\mathrm{p}$-i-n structure with different doping profiles ate source $(\mathrm{P}+)$, channel(i), and drain $(\mathrm{n}+)$ regions within the electrolytic solution along with a gate as the controller for the initial condition. Over the intrinsic channel region, a thin oxide layer is employed with the receptor to capture the target biomolecules. They classified the detection mechanism in two steps. The first step is carried out after capturing the biomolecules which develop surface potential due to the presence of ions in the electrolyte by electrostatic screening [14]. In the second step there is a change in the tunneling current of the device due to the development of surface potential under the gate (gating effect).
They formulated the sensitivity of the device without considering any noise [14] and variability [21] issues. But this work shows improved sensitivity and response time. The arrangement for the structure is illustrated in the Fig. 3a followed by the improved sensitivity with the concentration of biomolecules in Fig. 3b.

The performance of a TFET based biosensor depends on how effectively the gate controls the intrinsic channel. The electrolytic gate sensor doesn't give better control over the channel because of noise [14] and variability [22] issues. To overcome the above issue, in $2015 \mathrm{~A}$. Gao et al. comes with new device architecture which is CMOS compatible silicon nanowire-based TFET (SiNW-TFET) biosensor [5] by using "top-down" fabrication approach with a low-cost anisotropic self-etching technique via tetramethylammonium hydroxide 
Fig. 4 The schematic illustration of working of SiNW-TFET (a) In the off state the tunnel length is high and tunneling is not possible (b) when $\mathrm{V}_{\mathrm{G}}<0$ the BTBT is possible at source-channel junction and further the tunneling width decrease after interaction of biomolecules, $\mathbf{c}$ when $\mathrm{V}_{\mathrm{G}}>0$ now the drain-channel junction is tunnel junction which result in the ambipolar conduction [Fig. 2 [5]]

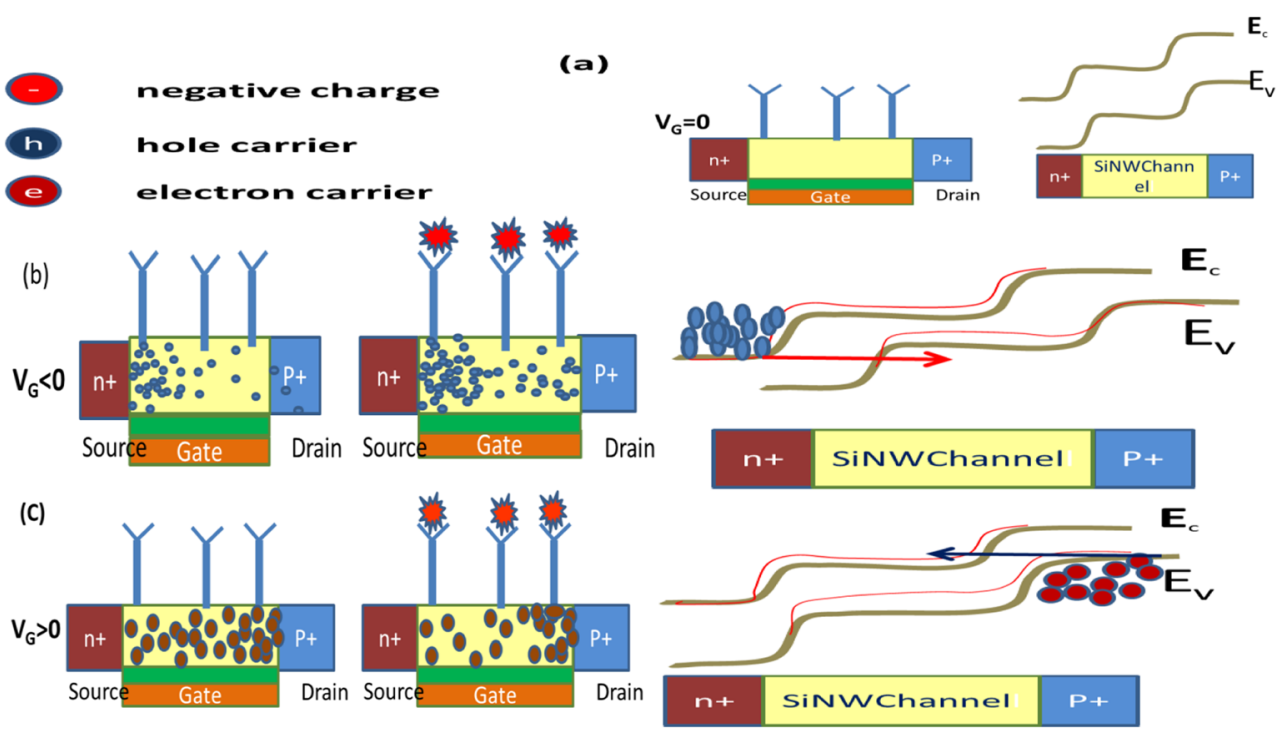

(TMAH). They implemented a planer gate structure over the nanowire channel for better control of the electrical conduction. Instead of using a single wire structure they grouped 10 nanowires into a single cluster and each wire used to detect biomolecules. The inclusion of ambipolar conduction is also taken as one of the parameters for the detection of both +ve and-ve charged biomolecules. With this kind of detection, the channel got duality nature and behaves either $n$-channel or $\mathrm{p}$ channel based on the detected charge biomolecules. The subthreshold swing for the device is reported as $37 \mathrm{mV} / \mathrm{dec}$ and $79 \mathrm{mV} / \mathrm{dec}$ for the $\mathrm{n}$-channel and P-channel TFET respectively at $300 \mathrm{~K}$ and the overall average SS for the device is reported as $76 \mathrm{mv} / \mathrm{dec}$ which is lesser than all other SiNW TFET biosensor[5]. The biomolecules are captured by the specific receptors functionalized on the surface of the SiNW FET. The process involved in the detection of the targeted biomolecule is illustrated completely with their energy band diagram in Fig. 4.

In the off state the device, the tunneling barrier width is high at both source and drain channel junction which is shown in Fig. 4(a). Figure 4(b) represents the energy band bending near the source and channel junction. The detection of -ve

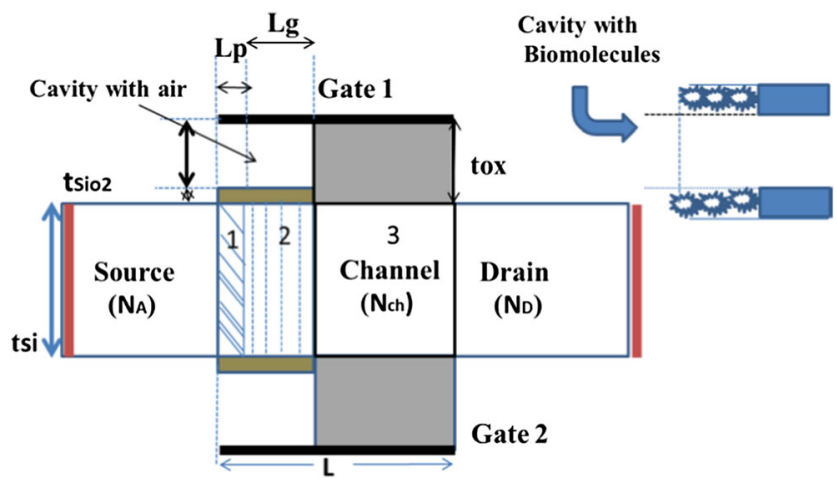

Fig. 5 Schematic view of the Dielectrically modulated TFET [Fig. 1[24]] charge carrier increases the barrier width which results in a decrease of ambipolar conduction as shown in Fig. 4(c). The red line in the energy band diagram indicates the bending in the bands from the initial state. They focused on the detection of the CYFRA21-1 by selecting a specific antibody on the SINW surface. CYFRA21-1 is a biomarker of human lung cancer. The proposed electronic biosensor not only improves the sensitivity but also able to distinguish the noise [14] from specific binding of a biomolecule by the uses of ambipolar conduction of TFET by revealing the signals form $\mathrm{P}-$ and $\mathrm{n}$ channel device.

\subsubsection{Dielectric Modulated TFET Based Biosensor}

The designing of label based biosensor is a very difficult and time- consuming process since utmost care has to be taken for the preparation of the bio-recognition/ sample element for the targeted biomolecules and the sample need to modify when the targeted analyte changed. The investigation of quality changes in the physiochemical reaction of the target analyte is also complicated and they are failing to detect the neutrally

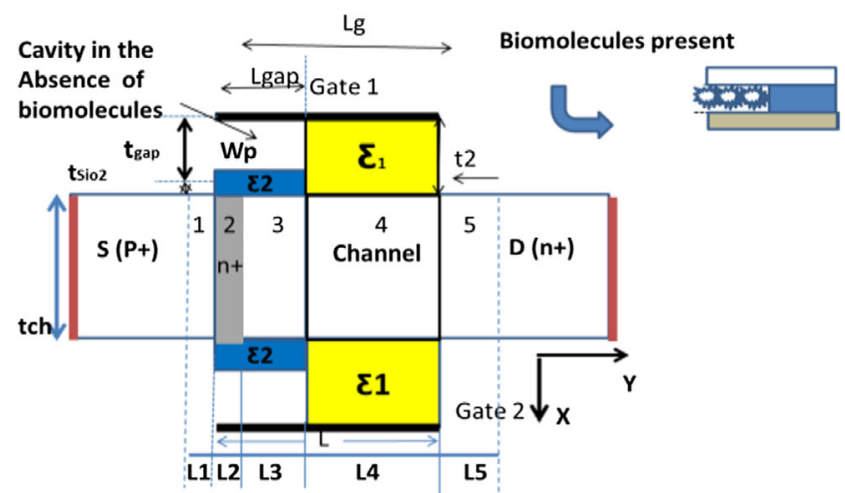

Fig. 6 Schematic view of the hetero gate dielectric of DMTFET [Fig. 1(a) [6]] 

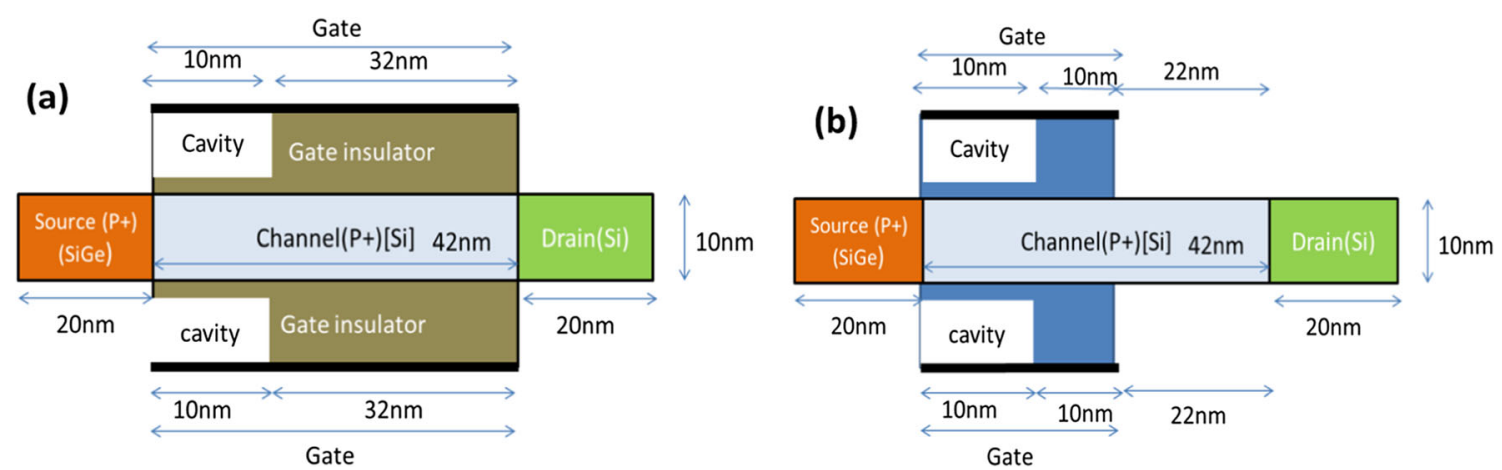

Fig. $7 \mathbf{a}$ and $\mathbf{b}$ the schematic view of the both full gate and the short gate DMTFET [Fig. 1(a) (b)[36]]

charged biomolecules. The Dielectric modulation technique [23] provided label-free detection of biomolecules Which suppress the challenges faced by the label-based detection techniques [5, 13].

R.Narang and et al. reported the first dielectric modulated TFET [24] biosensor is by utilizing the concept of dielectric modulated FET for biosensing [23]. The structure comes with a cavity region [25] where the targeted biomolecules are made to occupy and immobilize. The cavity region created in the oxide layer under the gate electrode. Once the biomolecules become stable and because of their dielectric value then the device experiences change in the dielectric constant in the oxide. Because of the change in dielectric constant the effective coupling between the gate and oxide layer changes in such a way that the energy bands of the channel start bending. This bending of the energy bands for channel results in the decrease/or increase in the effective tunneling length which
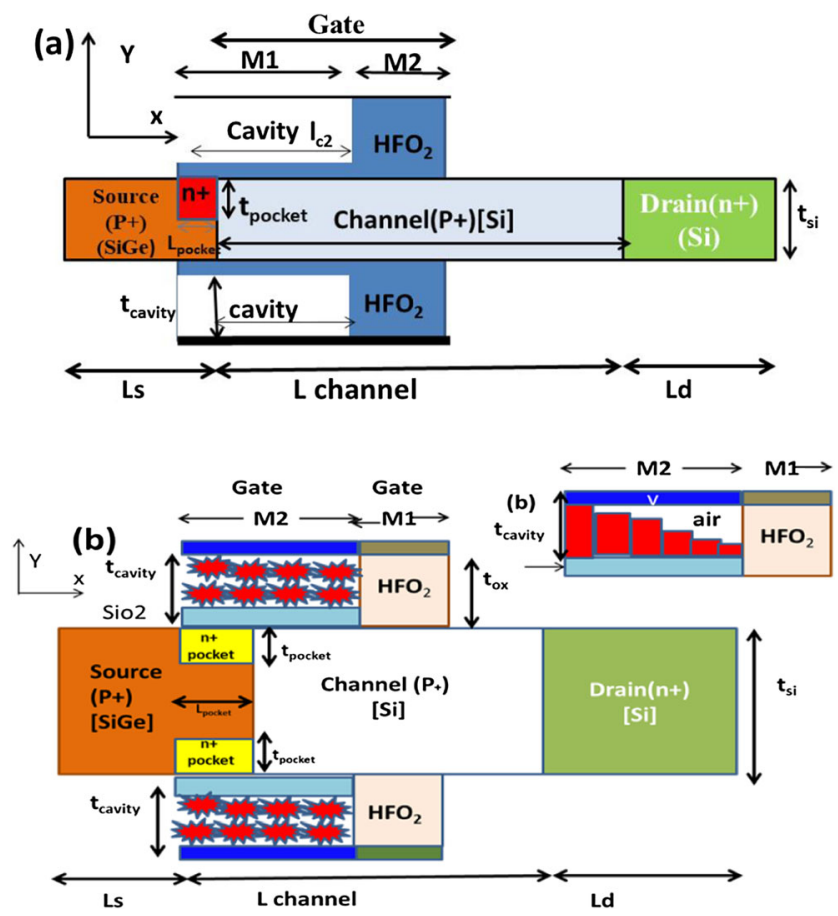

Fig. 8 a Schematic view of SGDMDMTFET [Fig. 1[40]] b Dual packet DPHTFET [41] leads to drift in the drain current. Instead of a single gate, a double gate structure is also considered because of their advantages described in $[26,27]$. Since the $p-i-n$ structure is having low on current, they considered the $p-n-p-n$ (Tunnel source MOSFET) as illustrated in Fig. 5.

The ambipolar conductivity is the most impediment character for TFET to improve performance towards sensitivity. The earlier reported dielectric modulated TFET biosensor [24] performance is limited because no care has been taken to reduce the ambipolar conductivity. The sensitivity analysis carried out by considering the charge and dielectric constant of the targeted biomolecules separately but practically the charge present only when the biomolecule present with a dielectric constant. To overcome these challenges and enhance the performance Rakhi Narang et al. proposed DMTFET [6] biosensor with a hetero gate structure. In this work, they carried the sensitivity analysis with the effect of charge at different dielectric constant values $[28,29]$. The hetero gate structure enhances the gate modulation at the source-channel (tunneling junction) by using high $\mathrm{K}$ value and low $\mathrm{K}$ value near the drain to reduce the ambipolar conduction[30]. The suppression of ambipolar conductivity improves the sensitivity toward both charge and dielectric effect. In the absence of biomolecule, it reported with low leakage current (10-17A/ $\mu \mathrm{m})$ which is lesser than the MOSFET.

The novel architecture of the proposed device is shown in Fig. 6 is a double-gated p-n-p-n architecture that contains dissimilar dielectric values $\mathrm{K} 1, \mathrm{~K} 2(\mathrm{~K} 1>\mathrm{K} 2)$ used for gate oxide to suppress the ambipolar conductivity[30] and increase the

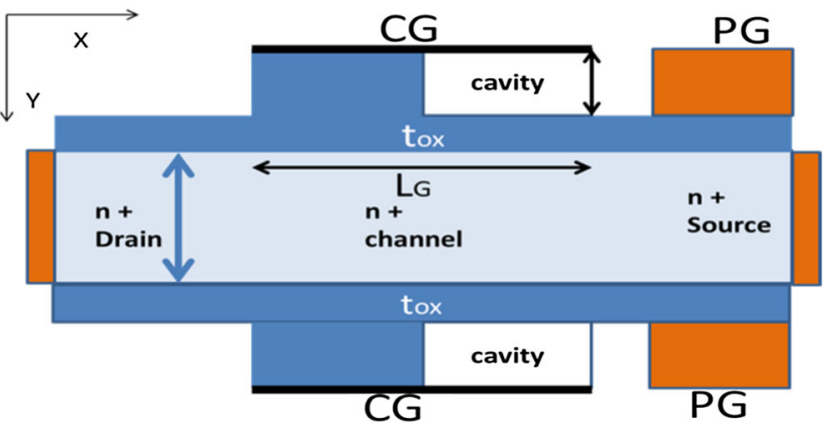

Fig. 9 Schematic arrangement of JL-DM-ED-TFET [Fig. 1[12]] 

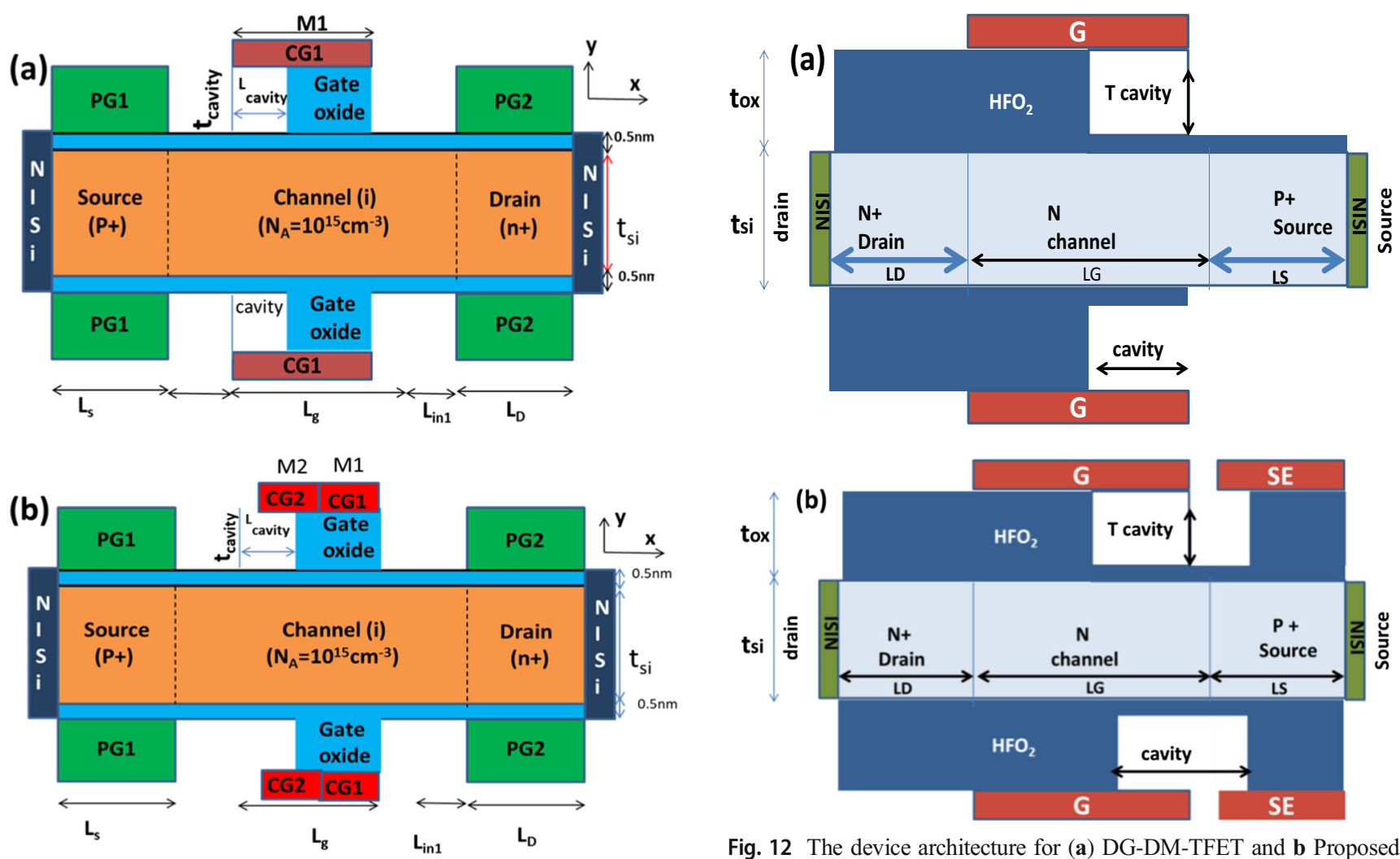

Fig. 10 a \& b schematic arrengement of SGDMTFET and SGDMDMTFET [44]

sensitivity. The cavity region is created near the tunneling junction of the device to immobilize targeted biomolecules.

The hetero gate DMTFET biosensor [6] reported high sensitivity by controlling the ambipolar conductivity but still this issue shows a considerable impact on device performance. The effective Scaling of gate length and structural modification suppresses the ambipolar conductivity [31-35] of the device to an extreme edge. The short gate structure of TFET (SG-TFET) [32] reported with high sensitivity with a low subthreshold swing by limiting ambipolar conductivity. In the year 2015 sayan kanungo et al. carried out the in-depth performance analysis of both short gate and full gate dielectrically modulated tunnel FET biosensors [36]. They completely givens the impact of structure modification in terms of energy band profiles and tunneling length at the

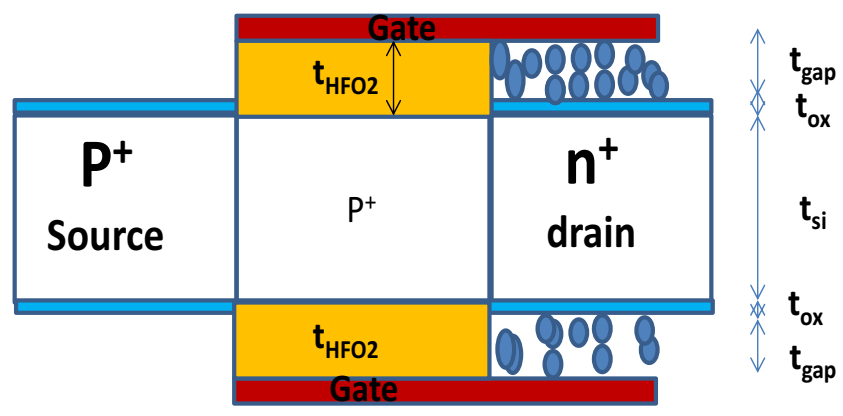

Fig. 11 Schematic view of structure [Fig i [21]]

Fig. 12 The device architecture for (a) DG-DM-TFET and b Proposed DG SE DM-TFET [(Fig. 1 [48]]
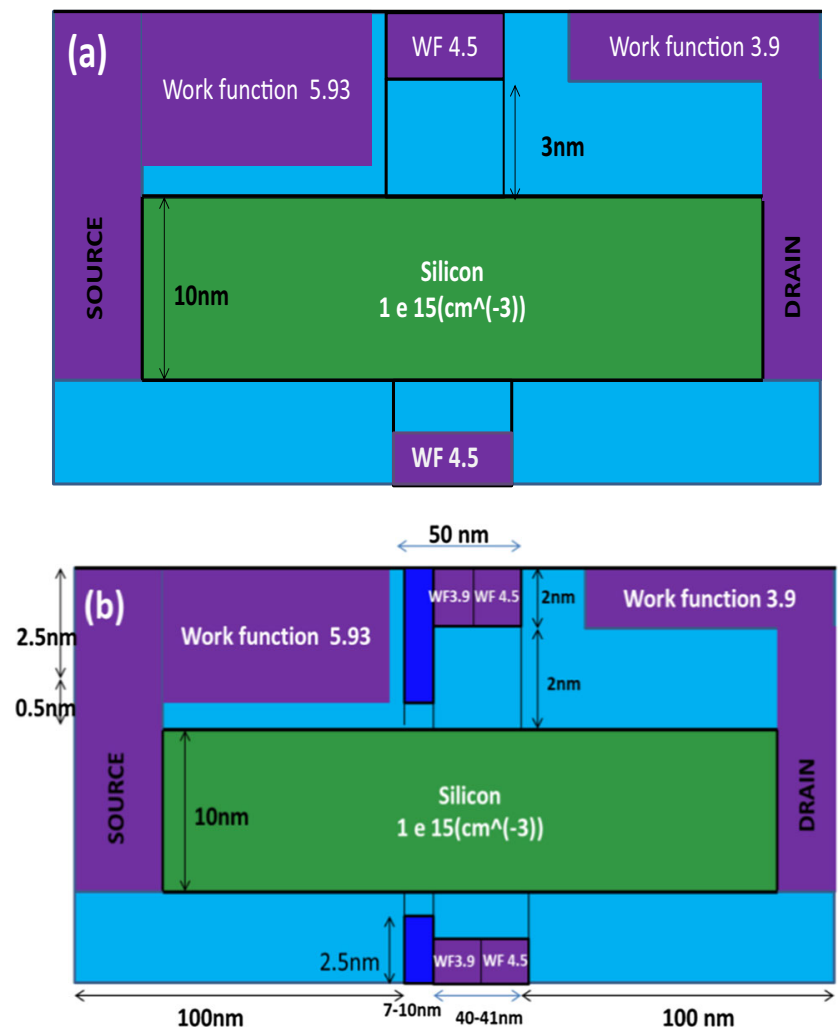

Fig. 13 a The schematic structure of conventional doping less TFET (b) charge plasma based gate underlap dielectrically modulated TFET [fig [49]] 


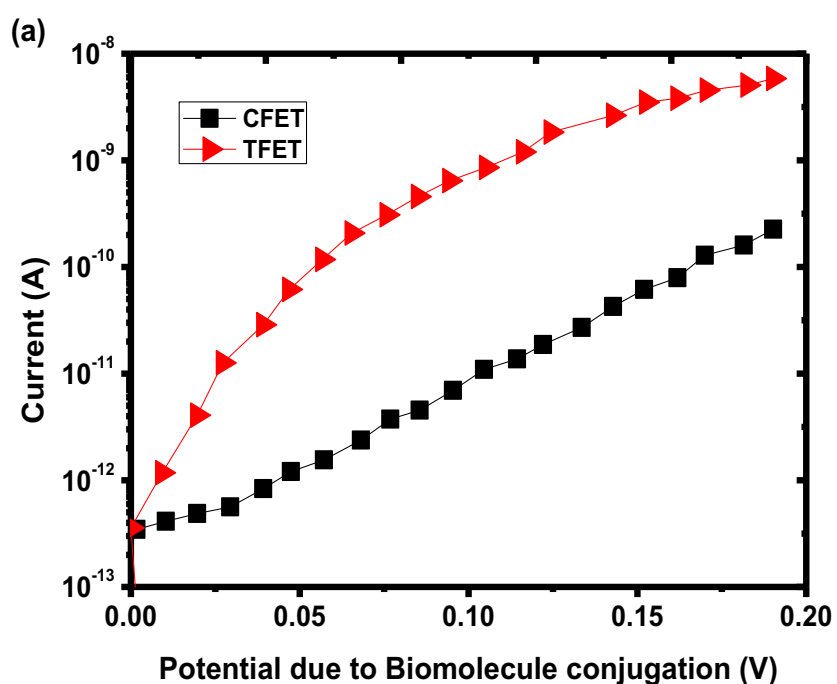

(b)

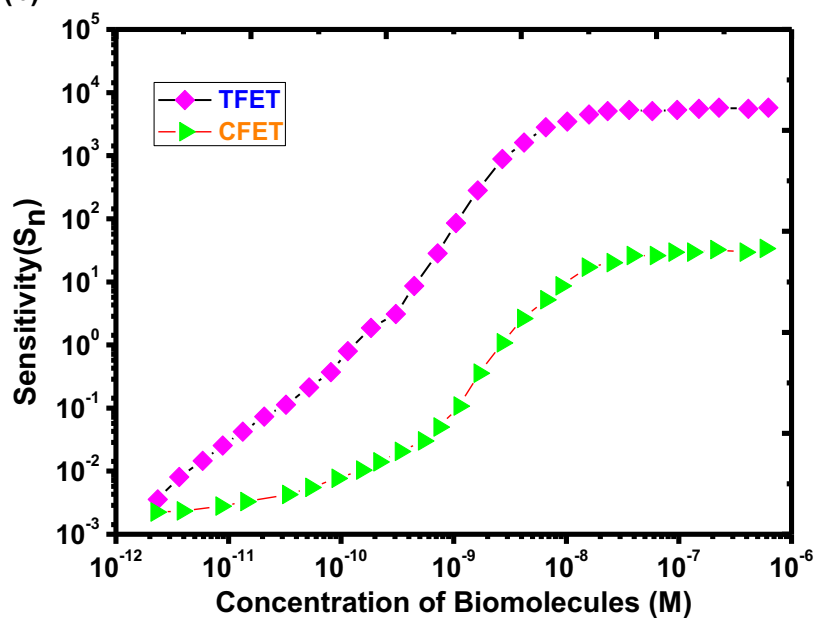

Fig. 14 a current variation as a potential developed due to biomolecules (b) sensitivity as function of biomolecules concentrations [Fig. $3(\mathrm{a}, \mathrm{c})[13]]$

junctions of source, channel, and drain. For the FG-DMTFET the intrinsic channel is completely gated but in the case of SGDMTFET the intrinsic channel is half gated. The presence of non-gated region near the drain in the SG-DMTFET the effective coupling between the gate and channel is reduced as a result the barrier width increases at drain-channel junctions. The increment in the effective barrier width decreases the tunneling current (ambipolar current) at the drain side. The tunneling current at the source-channel junction increases because of the initial gate to channel coupling thereby improving the sensitivity. The systematic arrangement of both SG-TFET and FG-TFET biosensor given in Fig. 7(a) and (b).

The on current (Ion) for dual metal SG-DMFET is limited because of one-directional tunneling (lateral tunneling) at the source-channel junction. This problem can be solved by vertical TFET which exhibits tunneling in two directions i.e. vertical (line tunneling) and lateral tunneling (point tunneling) [37-39]. By taking the above consideration, the first time

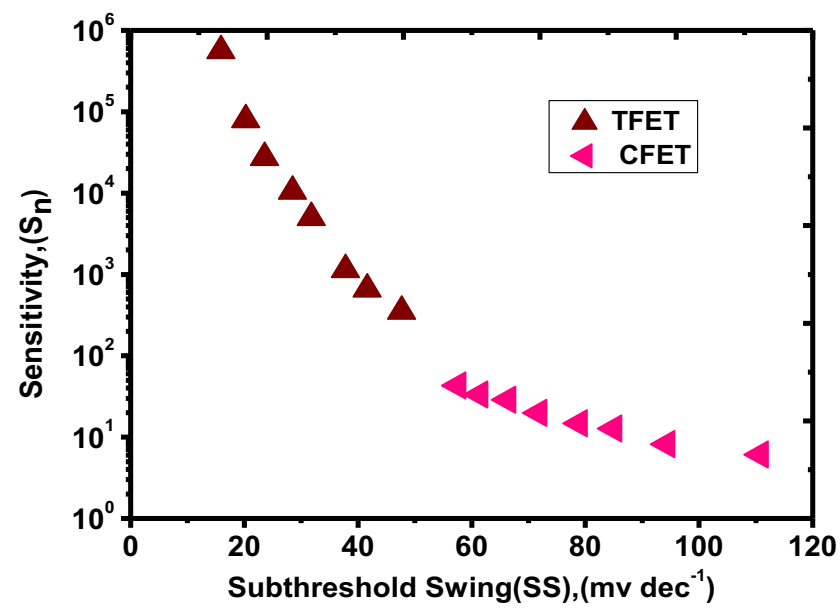

Fig. 15 a sensitivity as a function of sub threshold swing [Fig. 3(a,c)[13]]

evaluation of dual metal short gate vertical DMTFET (VDMTFET) is taken by placing an additional front gate $\mathrm{n}+$ pocket in the source region by Madhulika Verma et al. [40]. This improves the sensitivity irrespective of the position of bio
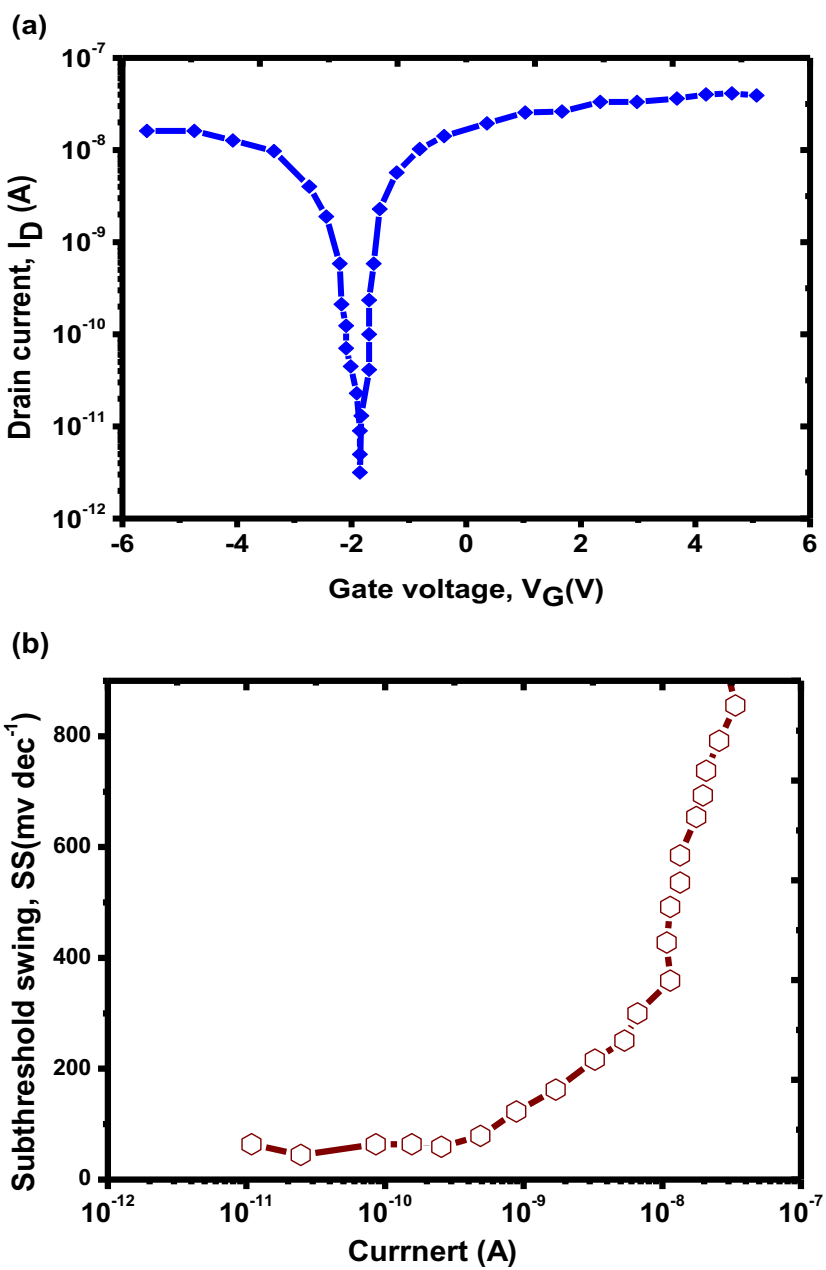

Fig. 16 a Id $-\mathrm{Vg}$ characteristics of SiNW-TFET for $\mathrm{v}_{\mathrm{D}}=1 \mathrm{~V}$ [fig. 1e[5]]. b sub threshold swing with current [Fig. 1e[5]]. for an n- channel $79 \mathrm{mv} /$ dec for P-channel and the average SS achieved is $76 \mathrm{mv} / \mathrm{dec}$. 
Fig. 17 Impact on sensitivity with increasing dielectric constant of both p-i-n and p-n-p-n TFET (b) impact of dielectric constant on the sensitivity for MOSFET and TFET [Fig. 3(b) and 2(C) of [24]]
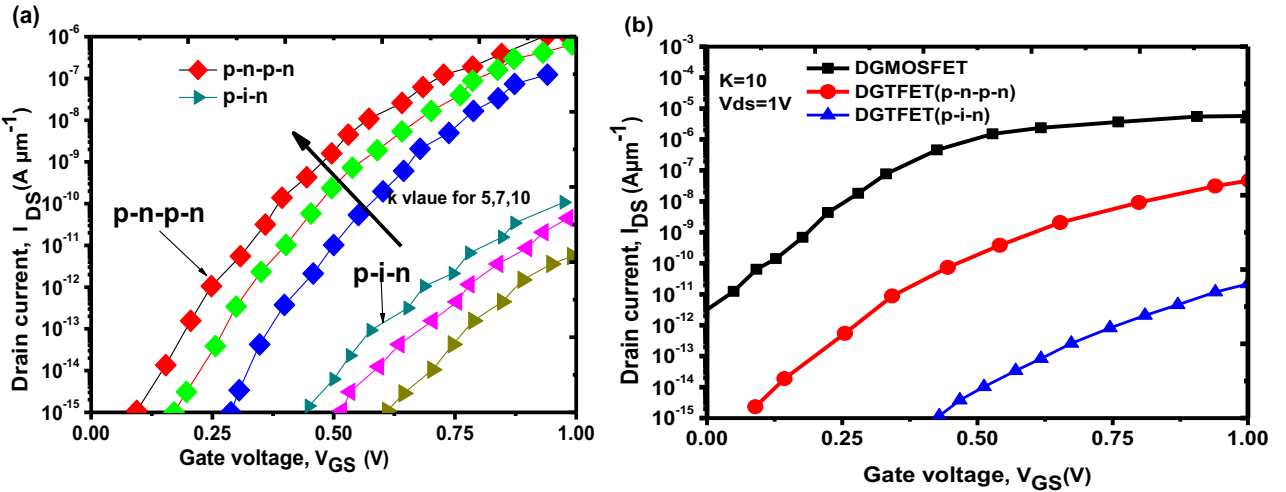

analyte inside the cavity. They compared the V-DMTFET with dual metal short gate DMTFET (L-DMFET) and the V-DMTFET reported with high on current and low subthreshold swing. The noise sensitivity of the V-DMTFET also has increased compared to the L-DMTFET sensor (Fig. 8) [41].

\subsubsection{Junction Less and Doping Less TFET Based Biosensors}

The performance of the TFET depends on the abrupt doping at source and channel junctions. The thermal annealing process is costlier and also it is very difficult to achieve uniform doping as well as thin junctions with physical doping. The random dopant fluctuation (RDF) [42] is another issue faced by the physically doped TFET based biosensor as reported earlier [6, $8-10,24,29,39,40]$. The Junction less TFET [11] is introduced to overcome the challenges faced by the means of physical doping. B. V. Chandan et al. proposed Junction less based dielectric modulated electrically doped TFET (JL-DM-EDTFET) biosensor for label-free detection of biomolecules [12].

The utilization of a control gate and polarity gate with suitable work function [43] over the intrinsic silicon avoids the need for physical doping and form the p-i-n structure. The cavity is created under the control gate for the immobilization of biomolecules to enable dielectric modulation. The absence of junction increases the device performance and the schematic arrangement of the biosensor is shown in Fig. 9.

The implementation of junction less TFET biosensor [12] with the method of doping less improves the device performance by eliminating issues like RDF etc. [42]. The issues related to fabrication complexity reduce but the ambipolar conductivity still a challenging issue. The structural modification is the one method to reduce the ambipolar conduction and the different work has been done which shows good results [31-36]. By considering the advantage of junction less and structural modification D. Sharma et al. proposed a Short gate dialectically modulated electrically doped TFET [SGDM-EDTFET] [44]. Compared to full gate dielectrically modulated and electrically doped TFET biosensor [FGDMEDTFET] the SGDM-EDTFET biosensor shows improved sensitivity (Fig. 10) [44].

For all the dielectric modulated TFET based biosensors, the ambipolar conduction is treated as a parameter which degrades their sensitivity. Different researchers suggested many approaches to minimize ambipolar conduction $[6,36,44]$. The introduction of dielectric modulated overlapping gate-ondrain TFET as a label-free biosensor by D. B. Abdi et al. made this ambipolar conduction an advantage for sensing biomolecules[45]. As the dielectric constant value changes, it increases the energy bandgap near the drain-channel junction which drives the significant reduction of ambipolar current. This variation in the current treated as the sensitivity parameter for biosensors. The negative charge has less impact compared to the dielectric constant of the targeted molecule so it is neglected. The systematic arrangement for biosensor is given in Fig. 11.
Fig. 18 a Impact of charge molecule on the Ion of TFET for a range of dielectric constant value (b) the impact of variation of Ion current of MOSFET with different $\mathrm{K}$ Values [Fig. 7.a and 8(b) of [24]]
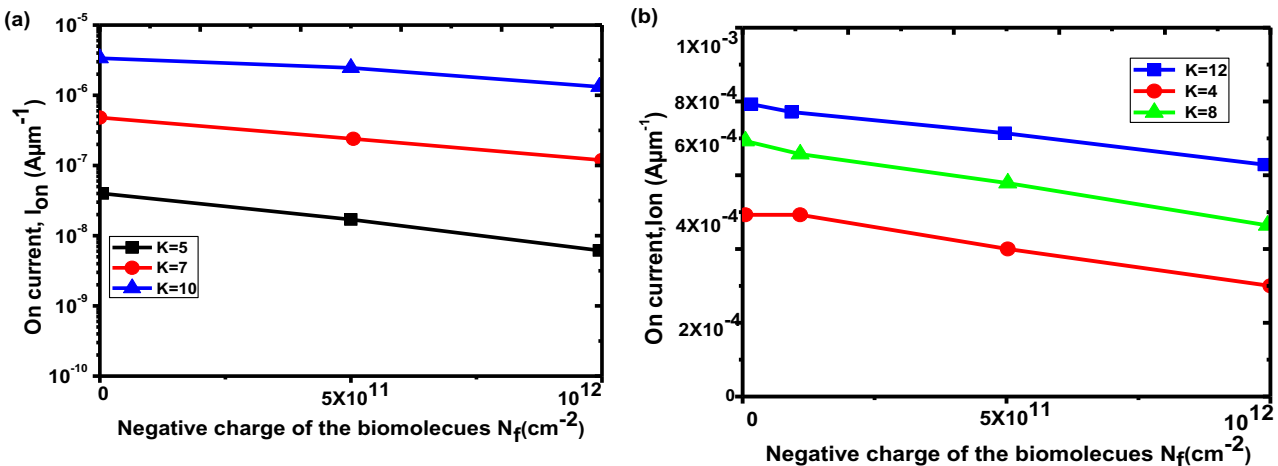


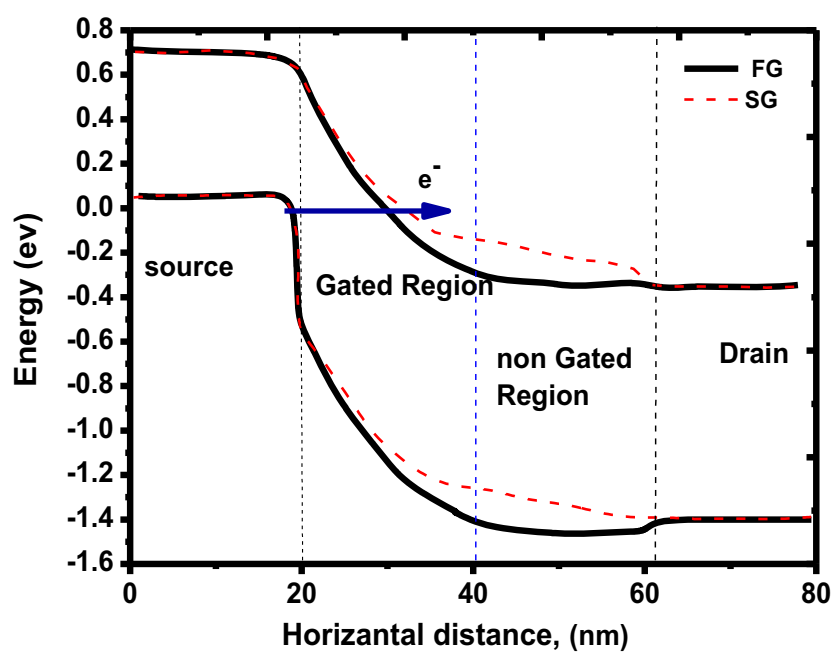

Fig. 19 Comparative energy profiles of FG and SG at [fig. 1(b) [36]]

\subsubsection{Charge Plasma Based TFET Biosensors}

The performance of a TFET depends on the achievable abruptness at the source-channel junction. This indirectly depends upon the doping profile of the device and because of the solubility limit of silicon it doesn't allow any further doping and is very tedious to create abrupt junction $[46,47]$ profiles. The charge plasma formation concept creates a solution for the formation of abruptness at the junction [46] in a simpler way. D. Soni et al. created this charged layer by placing an additional source electrode at the source side with a -ve voltage applied to it [48].

The device gives very good results of selective detection of biomolecules and shows high selectivity for specific volatile organic compounds. The cavity region under the gate extends towards the source region for enhancing abruptness at the source-channel junction. Figure 12 represents the structure of the charge plasma-based TFET as well as the normal DMTFET.

The charge plasma formation $[46,48]$ based TFET biosensor is enhancing the device performance by creating abrupt junctions. But they face a serious issue like RDF [42] and high thermal annealing budget due to the presence of physical doping in the device. Many researchers suggested the electrically doped TFET is the way to overcome the problems faced by physical doping [15]. By blending the advantages of charge plasma and doping less a novel architecture of charge plasma based gate underlap DMTFET is presented in [49]. The structure is given in the by Fig. 13. The p-i-n structure is achieved by placing metal with suitable work function over the intrinsic silicon layer. Because of the absence of the physical doping and presence of dual material gate the abruptness at the junction is created which results the device to overcome the short channel effects. The sensitivity analysis is done by varying the thickness of the cavity region underlap gate. With this device, we can achieve large sensitivity and the label-free detection and cost-effective fabrication which make the device superior to all others at present.

\subsubsection{Transition Metal Dichalcogenides (TMDs) Material Based TFET Biosensor}

In recent times, the flexible and stretchable electrons attracted more attention in various fields like medical and robotic due to its performance advancement. The silicon (si) based TFET biosensors are offering excellent performance, but due to the brittle nature, they failed in the case of mechanical flexibility. Various attempts are taken to overcome this problem with the approaches like wafer thinning [50] and ultrathin electronic layers by printing silicon nano wires [51] but they are very difficult to handle. The 2D materials become the potential alternate with their ultrathin and excellent electrical properties. In the year 2019 PK Dubey et al. come up with the Transition metal dichalcogenide material based TFET for label-free detection of biomolecules [52, 53]. The TDM TFET show excellent sensitivity with a steeper subthreshold value of $50 \mathrm{mv} / \mathrm{dec}$ for 5 decade change in the drain current and a sensitivity of 2.11 for $5 \mathrm{mv}$ change in gate voltage.
Fig. 20 a Comparative plot of output characteristics under dielectric constant variation [fig. 8(a) of [36]]. b Comparative plot of output characteristics under Charge density variation [fig. 8(b) of [36]]
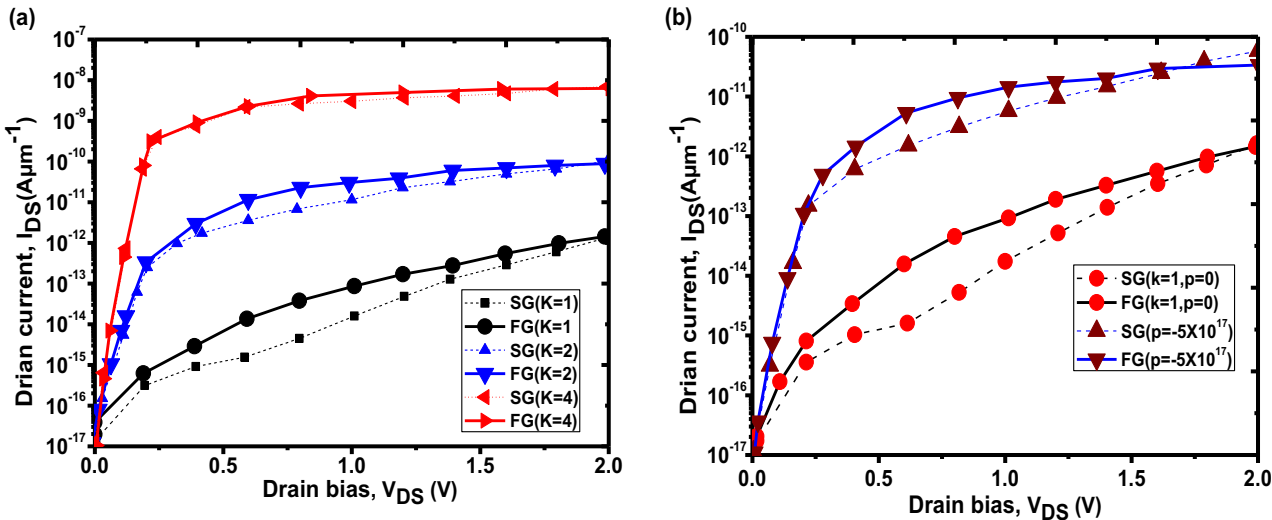
(a)

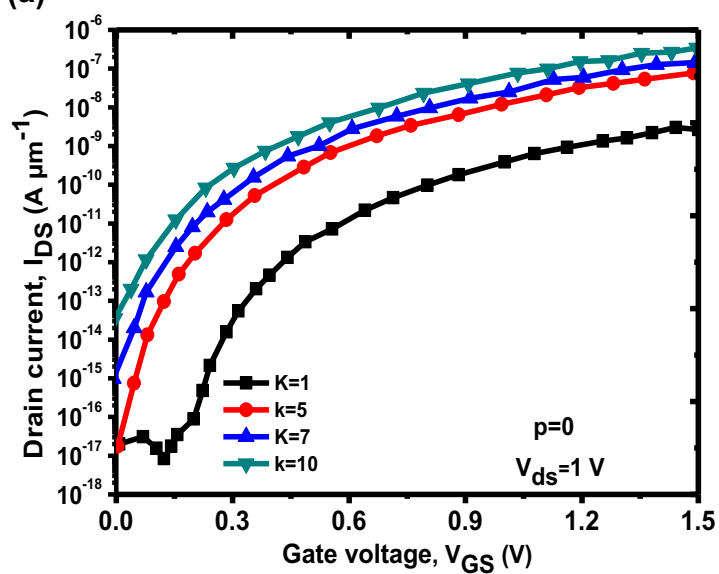

(C)

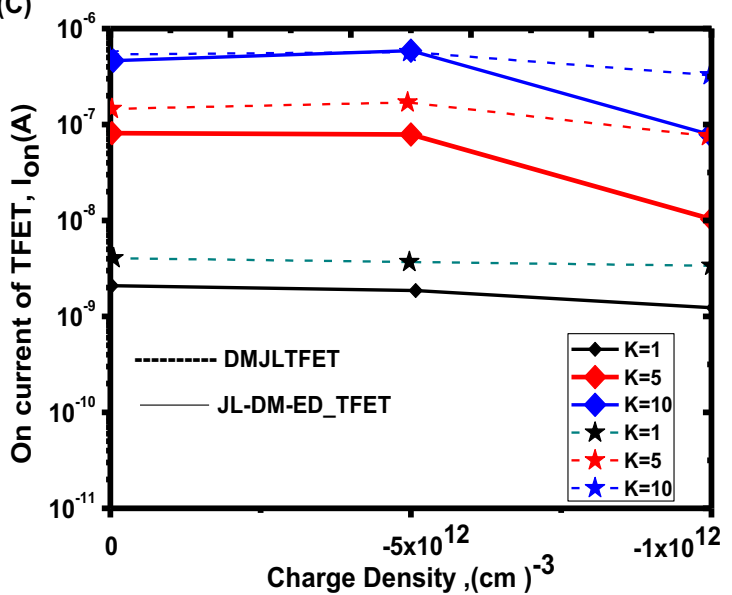

(b)

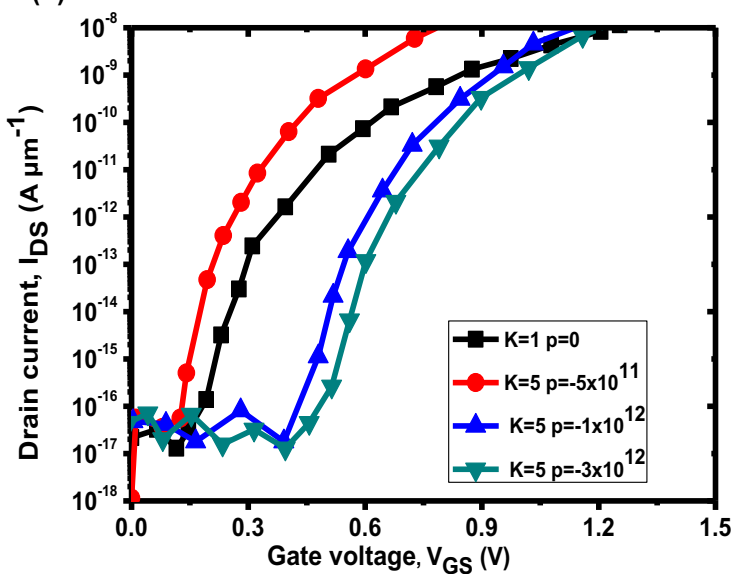

(D)

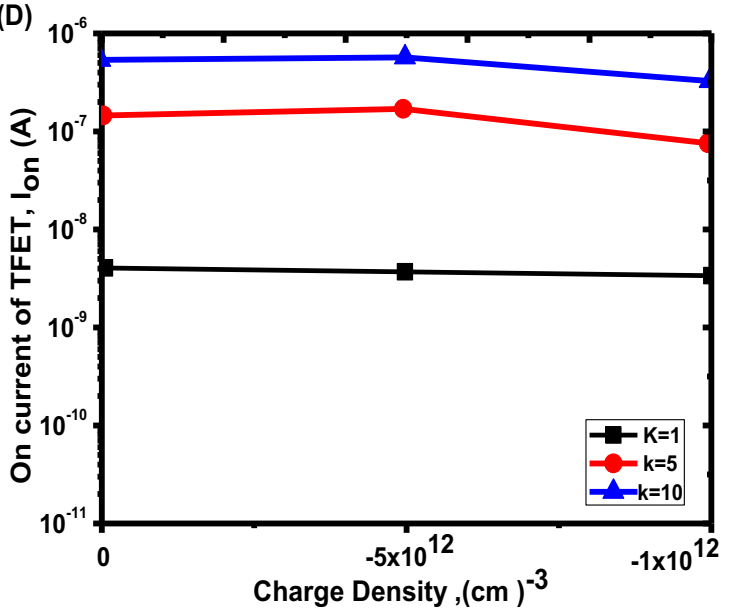

Fig. 21 a variation of drain current sensitivity with $\mathrm{K}$ values (b) variation of drain current sensitivity with charge density (c) \& (d) effect of charge density of the Ion current for [DMJLFET][JL-DM-ED-TFET] and MOSFET [fig. 4(a)(b) \& fig. 9(a)(b)(c)[12]]

\subsection{Performance Comparison with Respect to Sensitivity}

The sensitivity and selectivity are the two main factors that describe the performance of a biosensor. Form the above discussion it is observed that the change of physical structure and the detection mechanism improves the device performance.
The sensitivity of the TFET is measured by changes observed in the electrical property of the device before and after conjugation of the biomolecule with TFET. For the TFET sensitivity measured with the parameters such as the ratio of on current to the off current (Ion/Ioff), threshold voltage shift and the subthreshold swing, etc. Every individual formulated the sensitivity of the device by observing the variation of the (a)

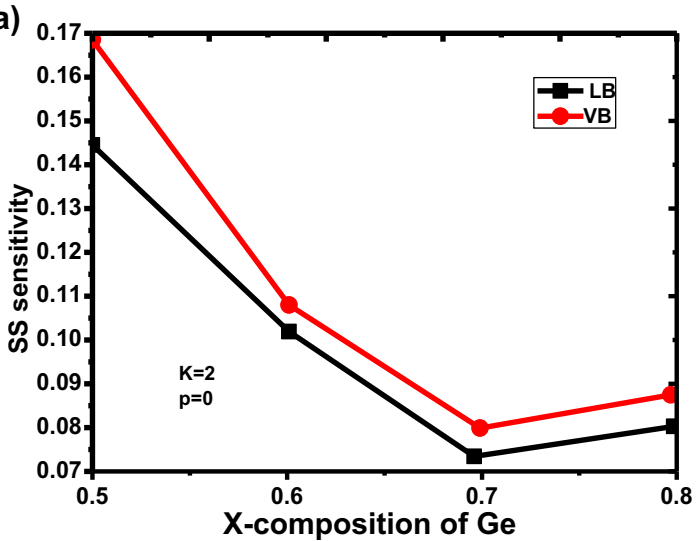

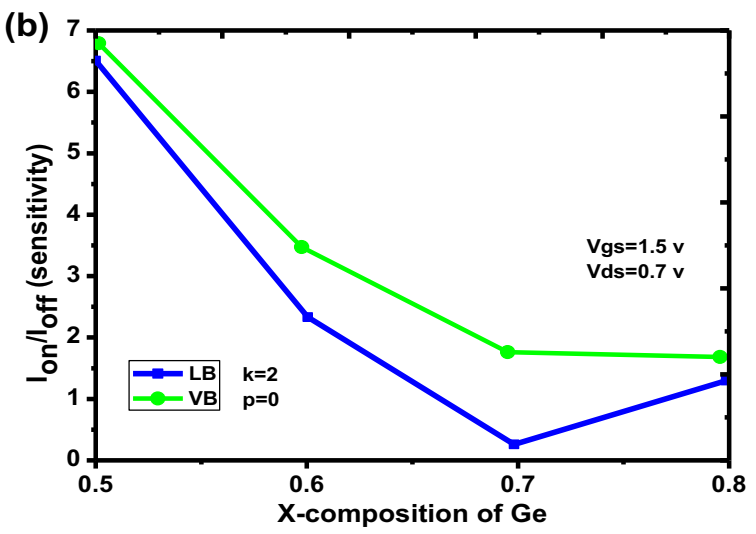

Fig. 22 a S S sensitivity and b Ion/Ioff sensitivity LB and VB along the X-composition of Ge [Fig. 9(a)(b) of [41]] 
Fig. 23 a Evaluation of sensitivity parameter DP-DMHTFET and SP-DM-HTFET with variation of thickness [fig. 4(a)[41]]. b Evaluation of sensitivity parameter DP-DMHTFET and SP-DM-HTFET with respect to the filling facto of LB,SP,DP [fig. 6(a) of the [41]] (a)

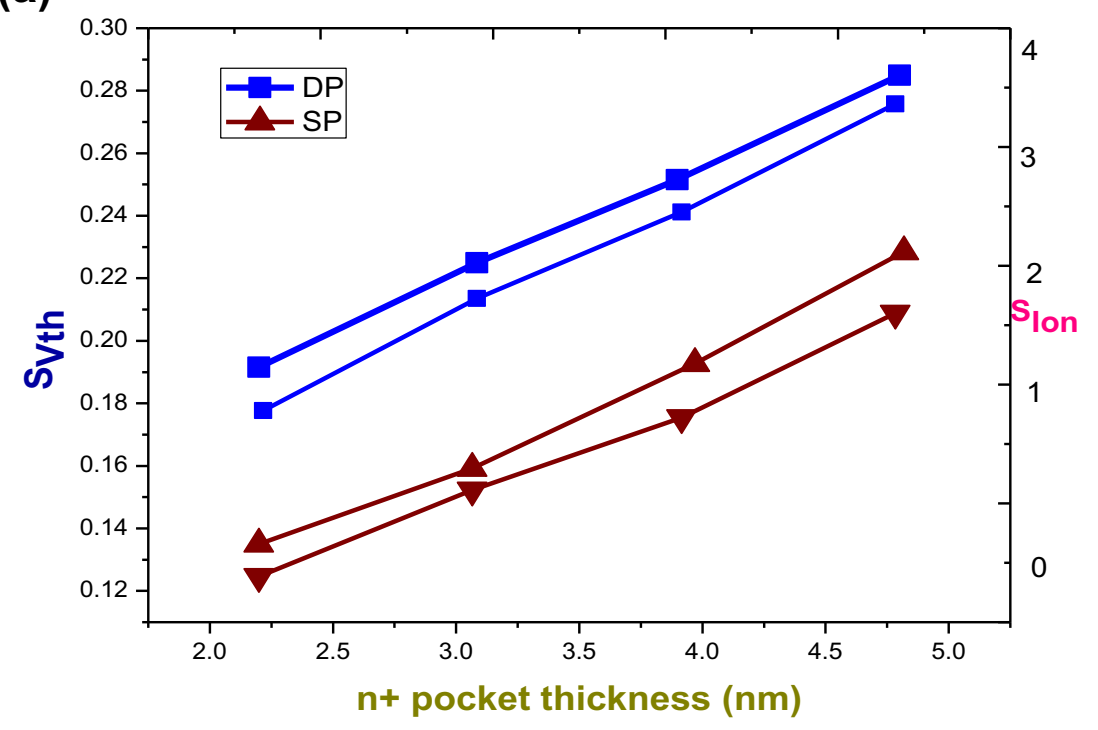

(b)

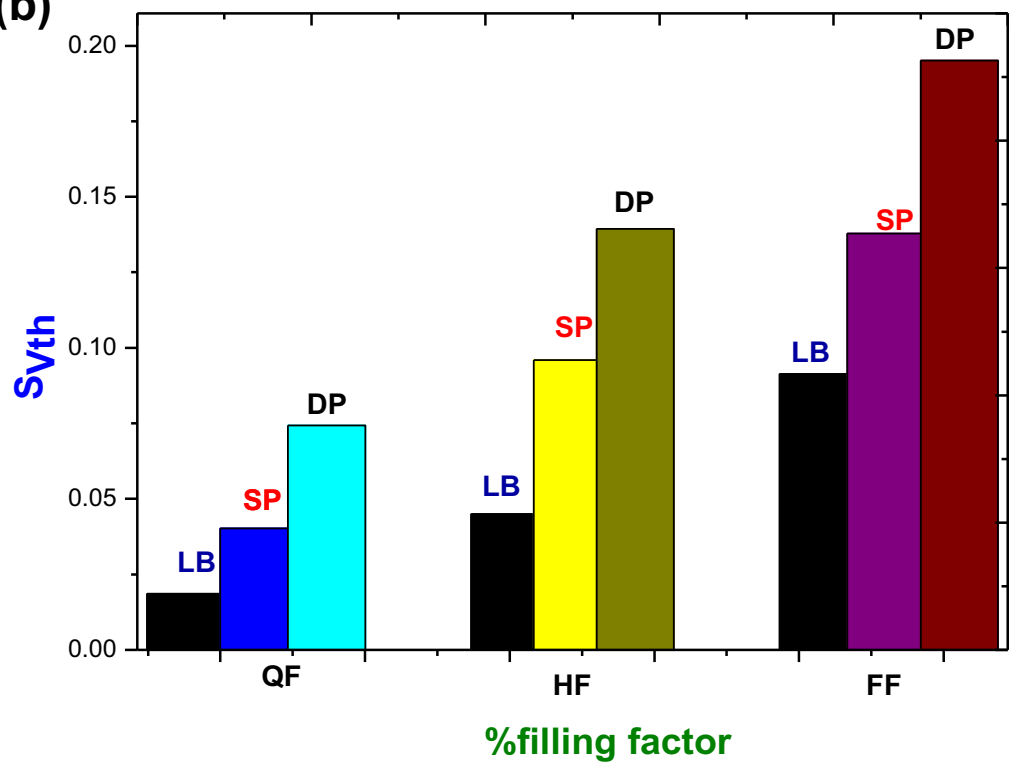

parameters. Here we carried the comparative analysis in sensitivity improvement from structure to structure. The first reported TFET based biosensor [13] produced high current than the CFET with the potential developed by the biomolecules as shown in Fig. 14.

It is observed the current as of the function of biomolecule conjunction and this will give the improved in the sensitivity of the biosensor because it produces high current for a small amount of potential given by the biomolecules. The subthreshold swing and the sensitivity plot of the structure [13] indicate that TFET can give the SS value less than the CFET shown in the Fig. 15.

For the point of ultra-low detection of biomolecules depends on the minimum achievable value for the device and this is achieved by the use of dual-channel with the bunch of nanowire TFET biosensors [5]. Figure 16 describe the current conduction for both positive and negative $(-v e)$ charge carrier and reported the high sensitivity with respect current change and achieved the minimum subthreshold swing of $37 \mathrm{mv} / \mathrm{dec}$.

The dielectric modulation provides the feasibility of detection with the variation of the dielectric constant of the biomolecules which gives the way of label-free detection and show the improvement in sensitivity because of the gating effect The sensitivity of the DMTFET can be compared concerning to the variation of two parameters.

(1) Variation of the dielectric constant and the charge of the biomolecule

(2) Variation of the geometry of the device.

Here they concentrated on the variation of dielectric constant and the charge of the biomolecules because the geometry 
(a)

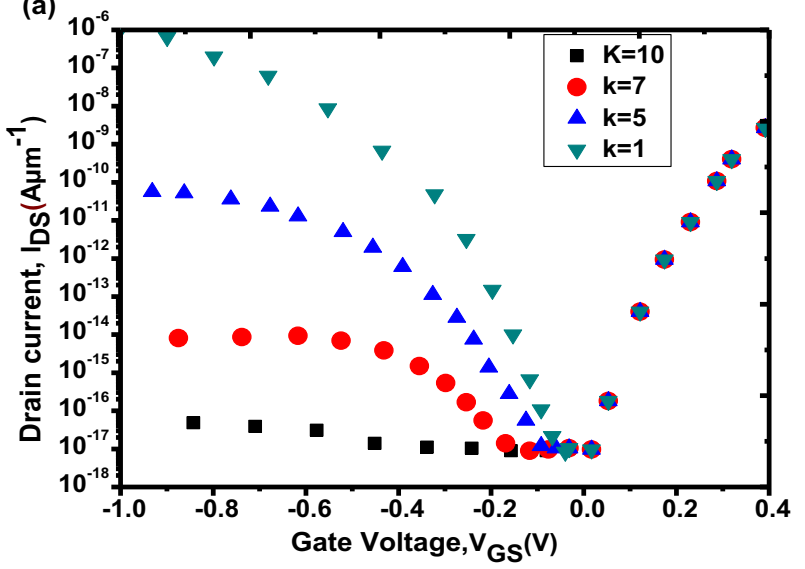

(b)

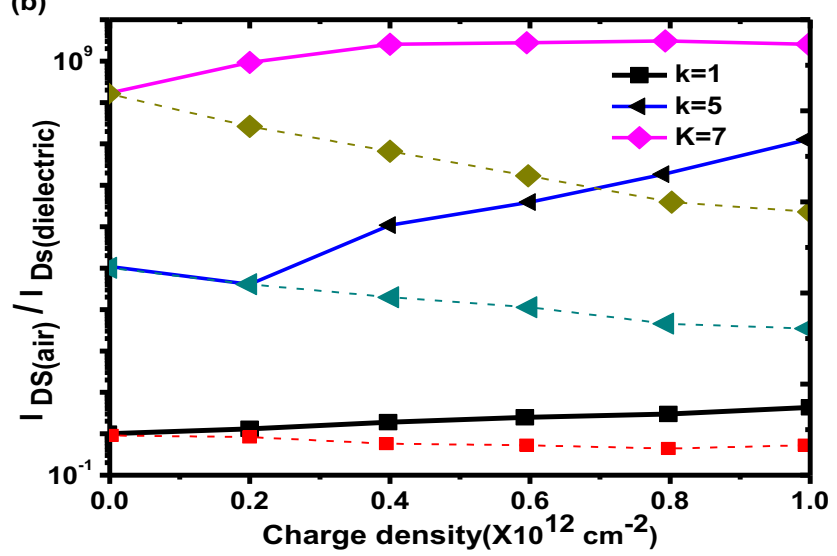

Fig. 24 a Transfer characteristics for different dielectric constants of the biomolecules immobilized in the nanogap (b) Drain current sensitivity variation of charge density and dielectric constant [45]

variation is having very little impact in the sensitivity of the device when compared with the Dielectric constant and charge variation so it is neglected.

The variation in the dielectric constant of the biomolecule along with charge makes the changes in the effective coupling between the gate and the channel which increases the sensitivity of the device. From Fig. 17 which shows that the.

p-n-p-n structure shows an enhanced on current (Ion) than the $\mathrm{p}$-i-n structure and the TFET is having low leakage current compared to MOSFET.

The impact of charges at different dielectric constants leads to the change in the Ion of the TFET and MOSFET which is shown in Fig.18. From the figure it evident that the TFET shows a higher impact on the on (Ion) current as compared to MOSFET.

The ambipolar behavior is suppressed by modification of the gate length [36] towards the drain side which widens the potential band gap near drain channel junction. The energy band diagram is shown in Fig. 19 which consists of both short gate and the full gate DMTFET.

The dotted line in the above Fig. 19 indicates the energy band of a short gate DMTFET where the controllability is
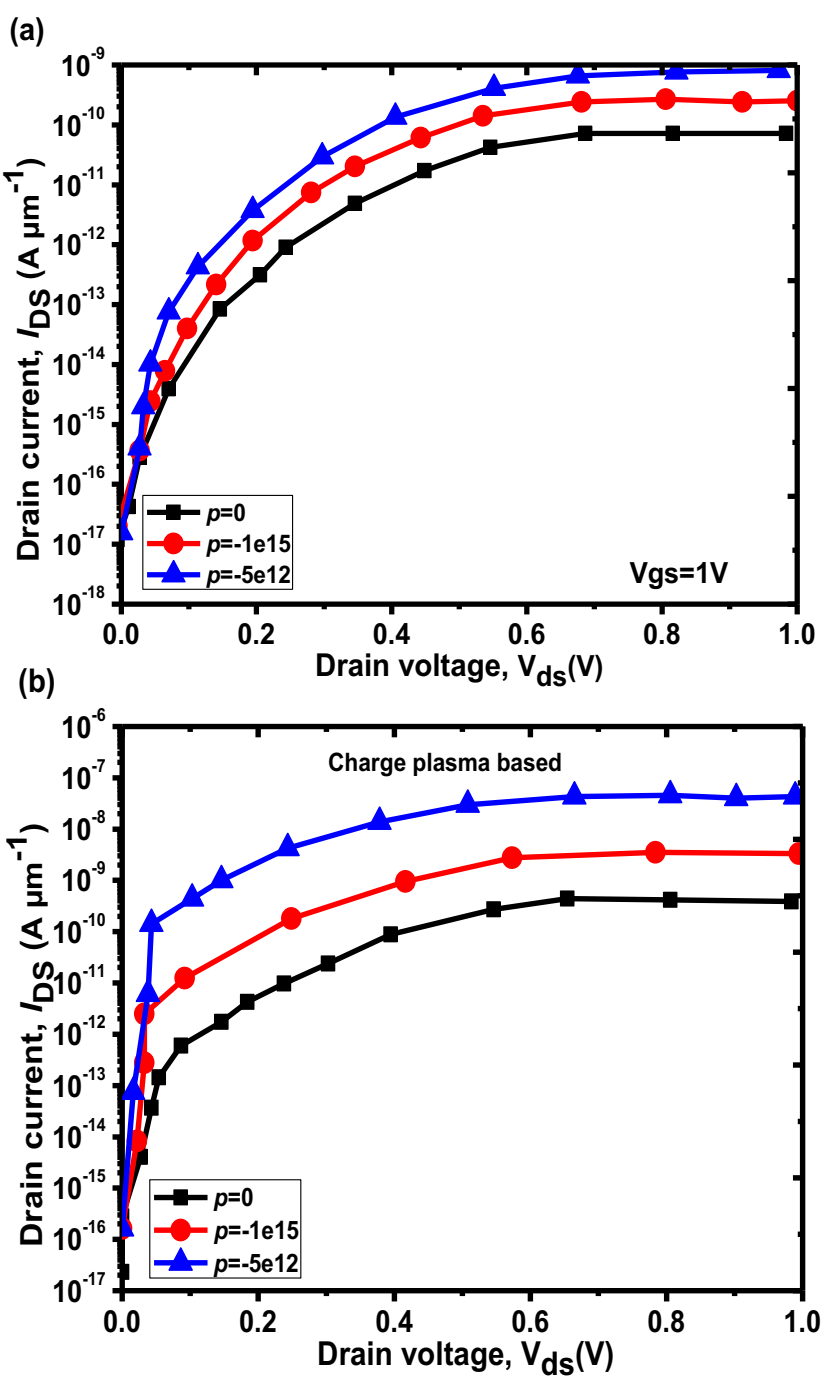

Fig. 25 a Drain current of the conventional TFET (b) the charge plasma based [48]

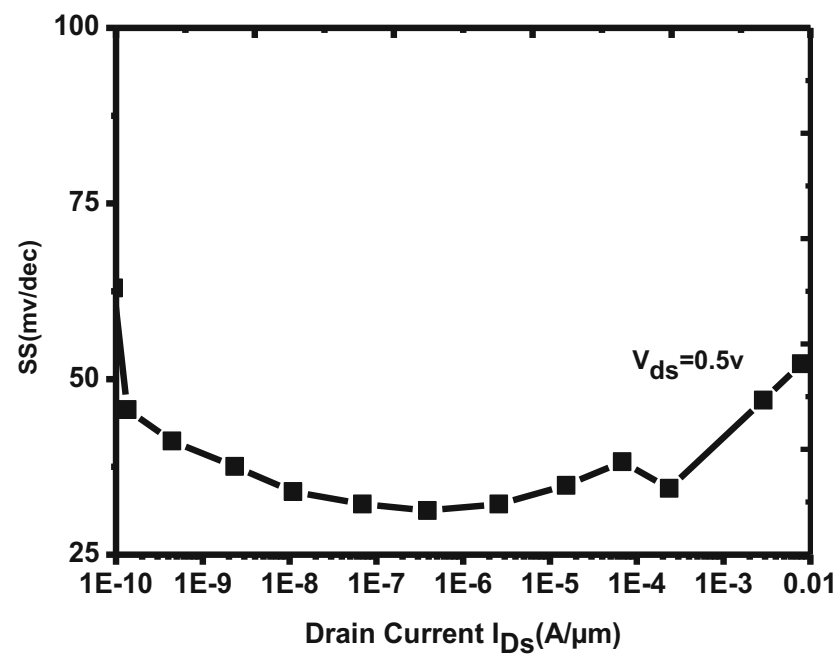

Fig. 26 The variation of the SS with respect to the decade change in the drain current [53] 


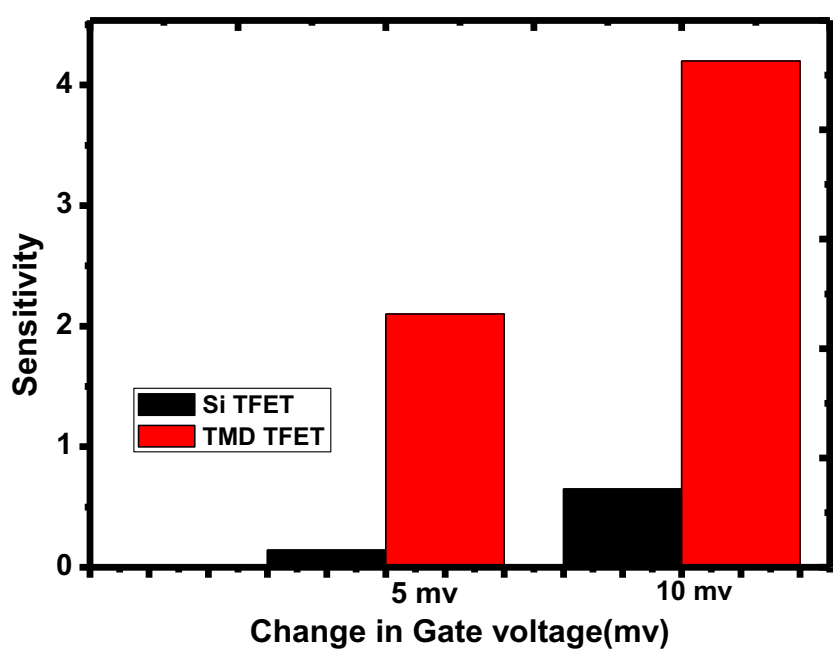

Fig. 27 The variation of the Sensitivity for the variation of gate voltage of silicon based TFET and the TMD based TFET [53]

achieved by reducing the effective coupling between the gate and the channel near the drain channel junction which decreases the ambipolar current.

From Fig. 20 it is observed that the full gate shows higher drain current than the Short gate since the drain biased induced gate control is weaker in the short gate by controlling the ambipolar conduction. The abruptness at the junction decides the tunneling of the charge carrier but it is difficult to create because of limitation. The JL-DMED-TFET transistor has made a breakthrough for the limitations. Figure 21 represents the variation in the drain current with different dielectric constant values and charge density and it is observed that the change of the $\mathrm{K}$ value shows an increase in the drain current to high values but on-state current decrease with increasing the charge density.

From Fig. 21 it is revealed that the JL-DM-ED-TFET has better on state current with the variation of charge density. The initial Ion current of silicon-based TFET is limited but this is solved by the introduction of vertical tunneling by placing a highly doped packet in the source under the gate overlap cavity [40].

From Fig. 22 it is evident that the vertical biosensor (VB) exhibits more sensitivity than the lateral biosensor (LB). The further enhancement in the sensitivity is achieved by the introduction of dual pockets in the source region and the improved sensitivity is reported [41]. The performance comparison of dual pocket is and the single pocket DM-TFET is compared with respect to the subthreshold swing. After observing the Fig. 23 it is observed that dual packet DP-DM-HTFET has higher sensitivity than SP-DM-HTFET and the lateral biosensor (LB) with improving the sensitivity.

The utilization of ambipolar current for detection results in the higher sensitivity of the device[45] as the dielectric constant of the device increase it reports a drastic change in decreasing the drain current which in turn improves the sensitivity of it is observed in the following Fig. 24.

The charge plasma improves the sensitivity by adding additional source electrode [6-12] which creates abruptness at the junction and the inclusion of these doping less with charge plasma concept enhances the device performance further by removing the physical doping drawback. The sensitivity comparison is shown in Fig. 25.

The conventional silicon material based TFET show excellent result but they are not able to fulfill forth the case of ultrathin size device because of the brittle nature of the silicon and recent times the flexible and stretchable electrons attracted more attention because of their excellent electrical properties. Transition metal dichalcogenide material based TFET biosensor proposed PK Dubey et al. [53] show the excellent control of the channel by the step subthreshold swing voltage show in the below Fig. 26. The sensor reports a very good sensitivity of 2.1 for $5 \mathrm{mv}$ change in the gate current which is very high when compared with other biosensors and shown in the Fig. 27.

From the above Figs. 26 and 27 it is clearly evident that the TMD based TFET biosensor are promising replacement for the future biosensors.

\section{Conclusion}

The striking advantages of TFET based biosensor over the conventional FET based biosensor in a real-time environment are presented along with performance metrics at a single point. The complete evaluation of TFET as a biosensor from the early stage to current improvement is presented with different approaches for the detection of biomolecules. From the comprehensive review it is concluded that the detection of biomolecules with the change of their dielectric constant is a good scope for future generation researchers. It is also concluded that doping less and the charge plasma formation concept eliminated the doping challenges and made the fabrication process is simple. It is also concluded that the vertical tunneling improves the tunneling by using two different possibilities like lateral and vertical tunneling. For measuring the sensitivity the ratio of on current to the off current(Ion/Ioff), the shift in the threshold voltage and subthreshold swing is considered but still there are many sensitivity parameters to be discovered which can be utilized for sensitivity improvement of TFET based Biosensor. Finally, it is concluded that the TFET device plays a crucial role for point- of -care application for getting accurate and reliable results because of its improved performance. 


\section{References}

1. Mehrotra P (2016) Science direct biosensors and their applications - A review. J Oral Biol Craniofacial Res 2015:1-7

2. Kougianos E (2006) Biosensors : A tutorial review. IEEE Potentials 25(April):35-40

3. Clark Jr CL, Lyons C (1962). Ann N Y Acad Sci 102:29

4. A. M. Villalba-rodr, R. Parra-saldivar, and H. M. N. Iqbal (2020) Electrochemical biosensors: a solution to Pollution Detection with Reference to Environmental Contaminants, pp. 1-21.

5. Gao A, Lu N, Wang Y, Li T (2016) Robust ultrasensitive tunneling- FET biosensor for point-of-care diagnostics. Sci Rep 6:22554

6. Narang R et al (2012) A dielectric-modulated tunnel-fet-based biosensor for label-free detection: analytical modeling study and sensitivity analysis. IEEE Trans Electron Devices 59(10):2809-2817

7. Sarkar D, Banerjee K (2012) Fundamental limitations of conventional-FET biosensors: Quantum-mechanical-tunneling to the rescue. Device Res Conf - Conf Dig DRC:83-84

8. Kanungo S, Chattopadhyay S, Gupta PS et al (2016) Study and analysis of the effects of SiGe source and pocket doped channel on sensing performance of dielectrically modulated tunnel FET based biosensor. IEEE Trans Electron Devices 63(6):2589-2596

9. Kanungo S, Chattopadhyay S, Gupta PS et al (2015) Comparative per- formance analysis of the dielectrically modulated full-gate and short- gate tunnel FET-based biosensors. IEEE Trans Electron Devices 62(3):994-1001

10. Narang R, Saxena M, Gupta M (2015) Comparative analysis ofdielectric- modulated FET and TFET based biosensor. IEEE Trans Nanotechnol 14(3):427-435

11. Ghosh B, Akram MW (2013) Junction less tunnel field-effect transistor. IEEE Electron Device Lett 34(5):584-586

12. Chandan VB, Nigam K, and Sharma D (2018) Junctionless based dielectric modulated electrically doped tunnel FET based biosensor for label-free detection vol. 13, pp. 452-456.

13. Sarkar D, Banerjee K (2012) Proposal for tunnel-field-effecttransistor as ultra-sensitive and label-free biosensors. Appl Phys Lett 100(14):143108

14. Nair PR, Alam MA (2008) Screening-limited response of nano biosensors. Nano Lett 8:1281-1285

15. Cao W et al. (2014) Subthreshold-swing physics of tunnel fieldeffect transistors Subthreshold-swing physics of tunnel field-effect, vol. 067141, pp. 0-9.

16. Wang PF, Hilsenbeck K, Nirschl T, Oswald M, Stepper C, Weis M, Schmitt-Landsiedel D, Hansch W (2004). Solid State Electron 48: 2281

17. Khatami Y, Banerjee K (2009). IEEE Trans Electron Dev 56:2752

18. Gandhi R, Chen Z, Singh N, Banerjee K, Lee S (2011). IEEE Electron Dev Lett 32:1504

19. Wadhera T, Kakkar D, Wadhwa G, Raj B (2019) Recent advances and progress in development of the field effect transistor biosensor: a review. J Electron Mater 48(12):7635-7646

20. Kumar MJ, Vishnoi R, Pandey P (2016) Tunnel field-effect transistors (TFET): modelling and simulation. Wiley, West Sussex

21. Shinwari MW, Shinwari MF, Deen MJ, Selvaganapathy PR (2011) Sensors and actuators B: Chemical The effect of DNA probe distribution on the reliability of label-free biosensors. Sensors Actuators B Chem 160(1):441-447

22. Deen MJ, et al. (2009) Noise considerations in field-effect biosensors Noise considerations in field-effect biosensors, vol. 074703, no. May 2014

23. Im H, Huang X, Gu B, and Choi Y (2007) LETTERS A dielectricmodulated field-effect transistor for biosensing, vol. 2, no. July, 2007.
24. Narang R, Saxena M, Gupta RS, Gupta M (2012) Dielectric modulated tunnel field-effect transistor-a biomolecule sensor. IEEE Electron Device Lett 33(2):266-268

25. Min KS, Kang CY, Park C, Park CS, Park BJ, Park JB, Hussain MM, Lee JC, Lee BH, Kirsch P, Tseng H-H, Jammy R, Yeom GY (2009) A novel damage-free high-k etch technique using neutral beam-assisted atomic layer etching (NBALE) for sub-32 nm technology node low power metal gate/high-k dielectric CMOSFETs. in IEDMTech Dig:435-438

26. Ahn J-H, Choi S-J, Han J-W, Park TJ, Lee SY, Choi Y-K (Aug. 2010) Double-gate nanowire field effect transistor for a biosensor. Nano Lett 10(8):2934-2938

27. Im M, Ahn J-H, Han J-W, Park TJ, Lee SY, Choi Y-K (Feb. 2011) De- velopment of a point-of-care testing platform with a nanogapembedded separated double-gate field effect transistor array and its readout system for detection of avian influenza. IEEE Sensors $\mathrm{J}$ 11(2):351-360

28. J. M. Kinsella and A. Ivanisevic, "Biosensing: Taking charge of biomolecules," Nat Nanotechnol, vol. 2, no. 10, pp. 596-597, Oct. 2007

29. Barbaro M, Bonfiglio A, Raffo L (2006) A charge-modulated FET for detection of biomolecular processes: conception, modeling, and simula- tion. IEEE Trans Electron Devices 53(1):158-166

30. Choi WY, Lee W (Sep. 2010) Hetero-gate-dielectric tunneling field-effect transistors. IEEE Trans Electron Devices 57(9):23172319

31. Verhulst AS, Vandenberghe WG, Maex K, Groeseneken G (2007) Tunnel field-effect transistor without gate-drain overlap. Appl Phys Lett 91(5):053102

32. Zhuge $J$ et al (2011) Digital-circuit analysis of short-gate tunnel FETs for low-voltage applications. Semicond Sci Technol 26(8): 085001

33. Mallik A, Chattopadhyay A (Dec. 2011) Drain-dependence of tunnel field- effect transistor characteristics: The role of the channel. IEEE Trans Electron Devices 58(12):4250-4257

34. Chattopadhyay A, Mallik A (Mar. 2011) Impact of a spacer dielectric and a gate overlap/underlap on the device performance of a tunnel field-effect transistor. IEEE Trans Electron Devices 58(3): 677-683

35. Jang J-S, Choi WY (Dec. 2011) Ambipolarity factor of tunneling field- effect transistors (TFETs). J Semicond Technol Sci 11(4): 272-277

36. Tunnel S, Biosensors F (2015) Comparative performance analysis of the dielectrically modulated full. IEEE Trans Electron Devices 62(3):994-1001

37. Lee H, Park J-D, Shin C (May 2016) Study of random variation in germanium-source vertical tunnel FET. IEEE Trans Electron Devices 63(5):1827-1834

38. Schmidt $\mathrm{M}$ et al (Jul. 2014) Line and point tunneling in scaled $\mathrm{Si} /$ SiGe heterostructure TFETs. IEEE Electron Device Lett 35(7):699_ 701

39. Ganapathi K, Yoon Y, Salahuddin S (2010) Analysis of InAs vertical and lateral band-to-band tunneling transistors: Leveraging vertical tunnelling for improved performance. Appl Phys Lett 97(3): Art. no. 033504. https://doi.org/10.1063/1.3466908

40. D. M. T. Biosensor, M. Verma, S. Tirkey, S. Yadav, D. Sharma, and D. S. Yadav (2017) Performance assessment of a novel vertical, vol. 64, no. 9, pp. 3841-3848.

41. Bhattacharyya A, Chanda M, De D (2019) Performance assessment of new dual-pocket vertical heterostructure tunnel FET-based biosensor considering steric hindrance issue. IEEE Trans Electron Devices: $1-6$

42. Leung G, Chui CO (2012) Variability impact of random dopant fluctuation on nanoscale junctionless FinFETs. IEEE Electron Device Letters 33(6):767-769 
43. Anand S, Amin SI, Sarin RK (2016) Performance analysis of charge plasma based dual electrode tunnel FET. J Semicond 37(5):054003

44. Sharma D, Singh D, Pandey S, Yadav S, Kondekar PN (2017) Comparative analysis of full-gate and short-gate dielectric modulated electrically doped Tunnel-FET based biosensors. Superlattice Microst 111:767-775

45. Abdi DB, Kumar MJ (2015) Superlattices and Microstructures Dielectric modulated overlapping gate-on-drain tunnel-FET as a label-free biosensor. Superlattice Microst 86:198-202

46. Jagadesh Kumar M, Nadda K (Apr. 2012) Bipolar charge-plasma transistor: a novel three terminal device. IEEE Transactions on Electron Devices 59(4)

47. Intekhab Amin S, Sarin RK (Sep. 2015) Analog performance investigation of gate misaligned double gate junctionless transistor. Journal of Computational Electronics, Springer 14(3):675-685

48. Soni D, Sharma D, Aslam M, Yadav S (2018) Approach for the improvement of sensitivity and sensing speed of TFET-based biosensor by using plasma formation concept. Micro Nano Lett 13(12): $1728-1733$
49. Wadhwa G (2018) Label free detection of biomolecules using charge-plasma- based gate underlap dielectric modulated. Junctionless TFET 47(8):4683-4693

50. W. T. Navaraj, S. Gupta, L. Lorenzelli, and R. Dahiya (Apr. 2018) Wafer scale transfer of ultrathin silicon chips on flexible substrates for high performance bendable systems. Adv Electron Mater, vol. 4, no. 4, Art. no. 1700277.

51. Núñez CG, Liu F, Navaraj WT, Christou A, Shakthivel D, Dahiya R (Dec. 2018) Heterogeneous integration of contact-printed semiconductor nanowires for high-performance devices on large areas. Microsyst Nanoeng 4(1):1-15

52. Dubey PK, Yogeswaran N, Liu F, Vilouras A, Kaushik BK, Dahiya R (2020) Monolayer MoSe-Based tunneling field effect transistor for ultrasensitive strain sensing. IEEE Trans Electron Devices

53. Dubey PK and Kaushik BK (2019) Transition metal dichalcogenide material based tunneling field-effect transistor for label free biosensing application.

Publisher's Note Springer Nature remains neutral with regard to jurisdictional claims in published maps and institutional affiliations. 